\title{
Non-circular motions and the cusp-core discrepancy in dwarf galaxies
}

\author{
J. van Eymeren ${ }^{1,2,3}$, C. Trachternach ${ }^{2}$, B. S. Koribalski ${ }^{3}$, and R.-J. Dettmar ${ }^{2}$ \\ 1 Jodrell Bank Centre for Astrophysics, School of Physics \& Astronomy, The University of Manchester, Alan Turing Building, \\ Oxford Road, Manchester, M13 9PL, UK \\ e-mail: Janine.VanEymeren@manchester .ac .uk \\ 2 Astronomisches Institut der Ruhr-Universität Bochum, Universitätsstraße 150, 44780 Bochum, Germany \\ 3 Australia Telescope National Facility, CSIRO, PO Box 76, Epping, NSW 1710, Australia
}

Received 20 March 2009 / Accepted 22 June 2009

\begin{abstract}
Context. The cusp-core discrepancy is one of the major problems in astrophysics. It results from comparing the observed mass distribution of galaxies with the predictions of cold dark matter simulations. The latter predict a cuspy density profile in the inner parts of galaxies, whereas observations of dwarf and low surface brightness galaxies show a constant-density core.

Aims. We want to determine the shape of the dark matter potential in the nuclear regions of a sample of six nearby irregular dwarf galaxies.

Methods. In order to quantify the amount of non-circular motions that could potentially affect a mass decomposition, we first perform a harmonic decomposition of the H I Hermite velocity fields of all sample galaxies. We then decompose the H I rotation curves into different mass components by fitting NFW and pseudo-isothermal halo models to the H I rotation curves using a $\chi^{2}$ minimisation. We model the minimum-disc, the minimum-disc + gas, and the maximum-disc cases.

Results. The non-circular motions are in all cases studied here of the order of only a few $\mathrm{km} \mathrm{s}^{-1}$ (generally corresponding to less than $25 \%$ of the local rotation velocity), which means that they do not significantly affect the rotation curves. The observed rotation curves can better be described by the cored pseudo-isothermal halo than by the NFW halo. The slopes of the dark matter density profiles confirm this result and are in good agreement with previous studies. The quality of the fits can often be improved when including baryons, which suggests that they contribute significantly to the inner part of the density profile of dwarf galaxies.
\end{abstract}

Key words. dark matter - galaxies: dwarf - kinematics and dynamics

\section{Introduction}

Early observations of spiral galaxies have shown that their dynamical mass, as inferred from their surprisingly flat rotation curves, cannot be explained by luminous matter alone (e.g., Bosma 1978; Rubin et al. 1978). Since then, "dark matter" has become part of modern cosmology. Currently, the most successful models use a cosmological constant $\Lambda$ as well as collisionless and dissipationless cold dark matter (CDM). $\Lambda$ CDM simulations, e.g., the Millennium Simulation by Springel et al. (2005) have been very successful in describing the observed large-scale structures in the Universe (Spergel et al. 2003, 2007), but they do not work properly on galaxy scales.

Next to the "missing satellite" problem (Moore et al. 1999), the "cusp-core" discrepancy (e.g., de Blok et al. 2001b) is still causing debate between observers and cosmologists. Numerical simulations predict cuspy haloes in the inner parts with a density distribution described by a power law $\rho(r) \sim r^{\alpha}$ with $\alpha$ ranging from -1 (e.g., Navarro et al. 1996) to -1.5 (e.g., Moore et al. 1998, 1999). This cusp leads to a steeply rising rotation curve. However, observations of dwarf and low surface brightness galaxies show that their rotation curves rise less steeply than predicted by CDM simulations (e.g., de Blok \& Bosma 2002). Similar conclusions have also been made from observations of high surface brightness disc galaxies (Salucci 2001). At small radii (typically a few kpcs), the mass distribution can better be described by a central, constant-density core (e.g.,
Flores \& Primack 1994; Côté et al. 2000; Marchesini et al. 2002; Gentile et al. 2004).

This interpretation has been met with skepticism by cosmologists. Thus, systematic effects in the data, like beam smearing, slit misplacement, slit width, and seeing, as well as the inclination of the galaxy and non-circular motions have been used to argue against the fact that the observations are incompatible with cusps (van den Bosch et al. 2000; Swaters et al. 2003; Hayashi et al. 2004; Spekkens et al. 2005). However, repeated one-dimensional long-slit spectra observed by independent observers with different telescopes (de Blok et al. 2003) as well as high-resolution $\mathrm{H} \alpha$ observations using a two-dimensional velocity field (Simon et al. 2005; Kuzio de Naray et al. 2006, 2008; Spano et al. 2008) rule out uncertainties related to slit spectroscopy and still suggest the presence of an isothermal dark matter core in the inner parts of disc galaxies. Non-circular motions can indeed affect the results of rotation curve studies, as it is generally assumed that the particles are on circular orbits. Several authors report that CDM haloes are triaxial objects with a globally elongated potential (e.g., Hayashi et al. 2004; Hayashi \& Navarro 2006). They suggest that this triaxiality induces large non-circular motions in the inner parts of galaxies (up to $15 \%$ of the maximum rotation velocity), leading to the observed cored profiles. Rhee et al. (2004) tried to quantify non-circular motions caused by bars, projection effects based on the thickness of the disc, and bulges, and found that they add up to a $30-50 \%$ 
Table 1. Some general properties of the sample galaxies.

\begin{tabular}{lcccc}
\hline \hline Galaxy & Type & \multicolumn{2}{c}{ Optical centre } & $D$ \\
& & $\alpha(\mathrm{J} 2000.0)$ & $\delta(\mathrm{J} 2000.0)$ & {$[\mathrm{Mpc}]$} \\
$(1)$ & $(2)$ & $(2)$ & $(2)$ & $(3)$ \\
\hline NGC 2366 & IB(s)m & $07^{\mathrm{h}} 28^{\mathrm{m}} 54.6^{\mathrm{s}}$ & $+69^{\circ} 12^{\prime} 57^{\prime \prime}$ & 3.44 \\
ESO 059-G001 & IB(s)m & $07^{\mathrm{h}} 31^{\mathrm{m}} 18.2^{\mathrm{s}}$ & $-68^{\circ} 11^{\prime} 17^{\prime \prime}$ & 4.57 \\
ESO 215-G009 & SAB(s)m & $10^{\mathrm{h}} 57^{\mathrm{m}} 29.9^{\mathrm{s}}$ & $-48^{\circ} 10^{\prime} 43^{\prime \prime}$ & 5.25 \\
NGC 4861 & SB(s)m & $12^{\mathrm{h}} 59^{\mathrm{m}} 02.3^{\mathrm{s}}$ & $+34^{\circ} 51^{\prime} 34^{\prime \prime}$ & 7.50 \\
NGC 5408 & IB(s)m & $14^{\mathrm{h}} 03^{\mathrm{m}} 20.9^{\mathrm{s}}$ & $-41^{\circ} 22^{\prime} 40^{\prime \prime}$ & 4.81 \\
IC 5152 & IA(s)m & $22^{\mathrm{h}} 02^{\mathrm{m}} 41.5^{\mathrm{s}}$ & $-51^{\circ} 17^{\prime} 47^{\prime \prime}$ & 2.07 \\
\hline
\end{tabular}

Notes: (1) The name of the galaxy; (2) data from NED; (3) distance references: NGC 2366: Tolstoy et al. (1995), ESO 059-G001: Karachentsev et al. (2006), ESO 215-G?009: Karachentsev et al. (2007), NGC 4861: de Vaucouleurs et al. (1991), NGC 5408: Karachentsev et al. (2002a), IC 5152: Karachentsev et al. (2002b).

Table 2. The main observational parameters.

\begin{tabular}{|c|c|c|c|c|c|c|c|}
\hline \multirow[t]{2}{*}{ Galaxy } & \multirow[t]{2}{*}{ Telescope } & \multirow[t]{2}{*}{ Arrays } & \multirow{2}{*}{$\begin{array}{c}\text { Spectral resolution } \\
{\left[\mathrm{km} \mathrm{s}^{-1}\right]}\end{array}$} & \multicolumn{2}{|c|}{ Synthesised beam } & \multirow{2}{*}{$\begin{array}{c}\text { Noise } \\
\text { myy beam }^{-1}\end{array}$} & \multirow[t]{2}{*}{ References } \\
\hline & & & & $" \times "$ & $\mathrm{pc} \times \mathrm{pc}$ & & \\
\hline NGC 2366 & VLA & $\mathrm{B}+\mathrm{C}+\mathrm{D}$ & 2.6 & $13 \times 12$ & $217 \times 200$ & 0.5 & (1) \\
\hline ESO059-G001 & ATCA & EW352+750C+1.5B & 4 & $51 \times 47$ & $1130 \times 1041$ & 1.3 & (2) \\
\hline ESO215-G?009 & ATCA & $\mathrm{EW} 352+750 \mathrm{~A}+1.5 \mathrm{C}+6 \mathrm{~A}$ & 4 & $21 \times 21$ & $530 \times 530$ & 1.0 & (2) \\
\hline NGC 4861 & VLA & $\mathrm{C}+\mathrm{D}$ & 5.2 & $31 \times 30$ & $1127 \times 1091$ & 0.6 & (3) \\
\hline NGC 5408 & ATCA & $375+750 \mathrm{D}+1.5 \mathrm{~A}$ & 4 & $57 \times 50$ & $1329 \times 1166$ & 1.9 & (4) \\
\hline IC 5152 & ATCA & $\mathrm{EW} 367+750 \mathrm{~A}+1.5 \mathrm{~A}$ & 4 & $50 \times 43$ & $502 \times 432$ & 1.5 & (2) \\
\hline
\end{tabular}

References: (1) Walter et al. (2008); (2) Koribalski et al. (2009); (3) van Eymeren et al. (2009b); (4) van Eymeren (2008).

underestimation of the rotation curve. However, de Blok et al. (2003) simulated rotation curves showing that non-circular motions of the order of $20 \mathrm{~km} \mathrm{~s}^{-1}$ over a large fraction of the disc are needed to make them consistent with CDM haloes. Furthermore, the observations by Gentile et al. (2005) and Trachternach et al. (2008) reveal that non-circular motions are typically of the order of a few $\mathrm{km} \mathrm{s}^{-1}$, i.e., too low to explain the cusp-core discrepancy. This might not always be the case, as Spekkens \& Sellwood (2007) found high non-circular motions in the spiral galaxy NGC 2976, which have a major effect on the rotation curve and which they explained by a bar in the inner $500 \mathrm{pc}$.

In this paper, the cusp-core discrepancy is addressed by using the rotation curves derived from H I synthesis data for a mass decomposition of a sample of six nearby irregular dwarf galaxies. The galaxies have been chosen to be a subsample of dwarf irregular galaxies in the Local Volume $(D<10 \mathrm{Mpc})$, which have been observed in H I with sufficiently high spatial resolution $(<1 \mathrm{kpc})$. Some general properties are given in Table 1.

In order to rule out systematic effects in the data, we first measure the non-circular motions. Non-circular motions can have two major causes: chaotic non-circular motions can be induced, for example, by star formation (Oh et al. 2008); systematic non-circular motions relate to the potential (e.g., spiral arms, triaxiality of the halo, see Schoenmakers et al. 1997). By performing a harmonic decomposition of the velocity fields, we are able to quantify the systematic non-circular motions.

This paper is organised as follows: in Sect. 2, the different data sets and the creation of the velocity fields are briefly described. The velocity fields and rotation curves are presented in Sect. 3. In Sect. 4, we describe the procedure of the harmonic decomposition and show and discuss our results. Section 5 presents the theoretical background of the mass modelling, which is followed by a discussion of the results from the mass decomposition in Sect. 6. In Sect. 7, the main results are summarised.

\section{Observations and data reduction}

For this study we selected six nearby irregular dwarf galaxies. Some observational details are given in Table 2.

H I data for the two northern galaxies, NGC 2366 and NGC 4861, were obtained with the Very Large Array (VLA). The IB(s)m galaxy NGC 2366 forms part of "The H I Nearby Galaxy Survey" (THINGS, Walter et al. 2008). Its peculiar kinematics were the subject of several recent publications (de Blok et al. 2008; Oh et al. 2008; Trachternach et al. 2008; van Eymeren et al. 2009a, hereafter dB08, Oh08, CT08, and vE09a). The SB(s)m galaxy NGC 4861 was studied by Wilcots et al. (1996) and Thuan et al. (2004). A detailed kinematic analysis of this galaxy by van Eymeren et al. (2009b) is based on the combined $\mathrm{H}$ I data of two $\mathrm{C}$ array and one D array observation. The data reduction process of NGC 2366 is described in Walter et al. (2008), that of NGC 4861 in van Eymeren et al. (2009b).

The four southern dwarf galaxies, ESO 059-G001, ESO 215G?009, NGC 5408, and IC 5152, are selected from the "Local Volume HI Survey" (LVHIS ${ }^{1}$; Koribalski \& Jerjen 2008; Koribalski et al. 2009). H I data for these galaxies were obtained with the Australia Telescope Compact Array (ATCA) and reduced by the LVHIS team (for details see Koribalski et al. 2009). All galaxies were observed for $12 \mathrm{~h}$ each in at least three different configurations (see Table 2).

For the purpose of this paper, H I velocity fields are created from the naturally weighted data cubes by fitting GaussHermite h3 polynomials to all line profiles using the GIPSY ${ }^{2}$ (The Groningen Image Processing System; van der Hulst et al. 1992) task xgaufit. This method allows us to accurately define the peak velocities, whereas the standard intensity-weighted

\footnotetext{
${ }^{1}$ LVHIS project webpage: www.atnf.csiro.au/research/ LVHIS/

${ }^{2}$ URL: http://www.astro.rug.nl/ gipsy/
} 

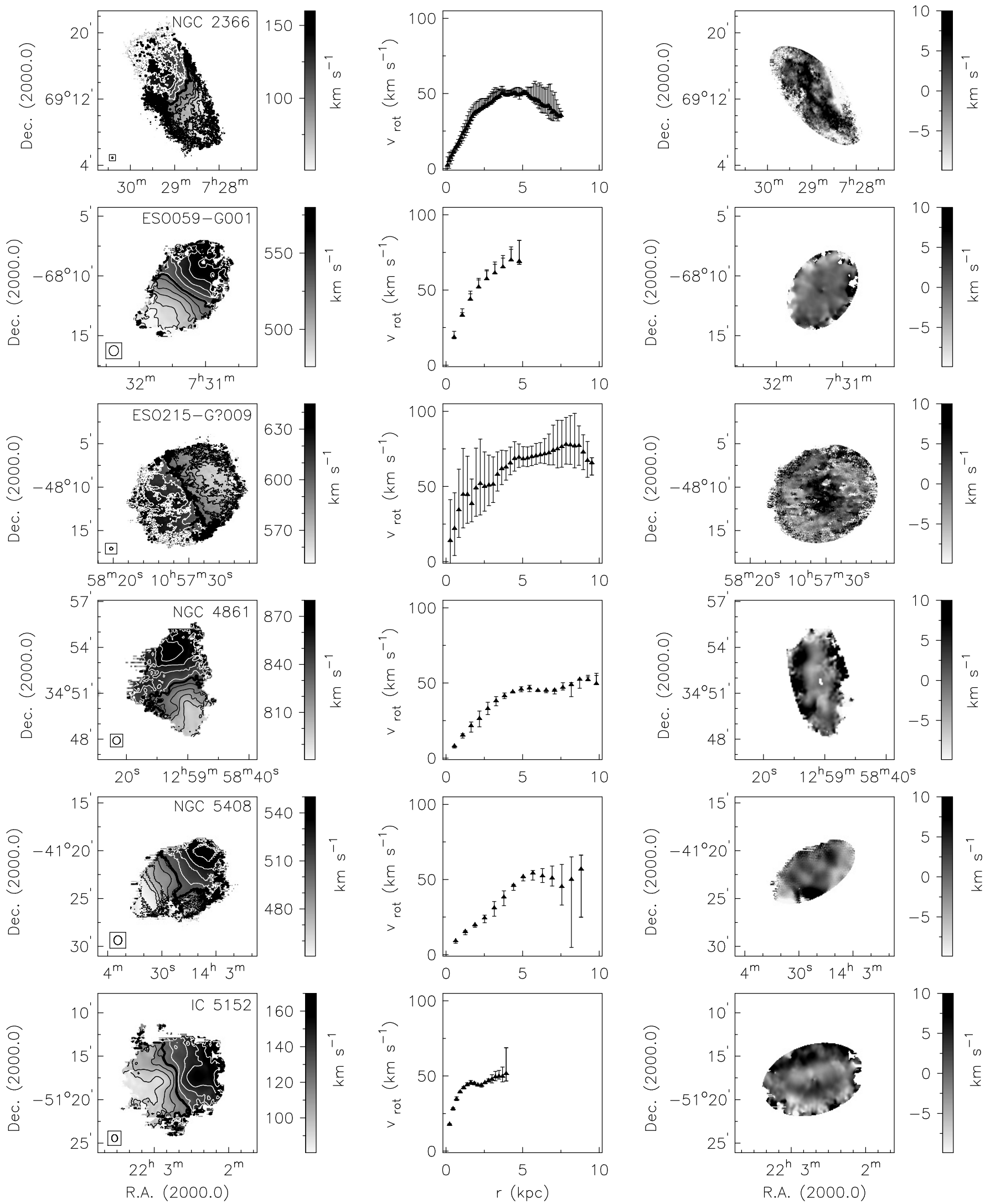

Fig. 1. The H I kinematics of all sample galaxies. Left panels: Hermite h3 velocity fields. The systemic velocity is marked by a bold line, the beam is placed in the lower left corner. Contour levels are overlaid in steps of $10 \mathrm{~km} \mathrm{~s}^{-1}$. Middle panels: the rotation curves derived from a tilted-ring analysis (see Table 3 for the fit parameters). Receding and approaching side are indicated by error bars. Right panels: the residual maps after subtracting a model velocity field (created from the fit parameters in Table 3) from the observed velocity field. 
Table 3. Kinematic parameters of the sample galaxies.

\begin{tabular}{|c|c|c|c|c|c|c|c|c|}
\hline \multirow{3}{*}{$\begin{array}{l}\text { Galaxy } \\
\text { (1) }\end{array}$} & \multicolumn{2}{|c|}{ Dynamic centre } & \multirow{3}{*}{$\begin{array}{c}v_{\text {sys }} \\
{\left[\mathrm{km} \mathrm{s}^{-1}\right]} \\
(2)\end{array}$} & \multirow{3}{*}{$\begin{array}{c}v_{\text {rot }} \\
{\left[\mathrm{km} \mathrm{s}^{-1}\right]} \\
(2)\end{array}$} & \multirow{3}{*}{$\begin{array}{c}i \\
{\left[{ }^{\circ}\right]} \\
(2) \\
\end{array}$} & \multirow{3}{*}{$\begin{array}{l}P A \\
{\left[{ }^{\circ}\right]} \\
(2) \\
\end{array}$} & \multirow{3}{*}{$\begin{array}{c}\alpha_{\text {opt }}-\alpha_{\text {dyn }} \\
{\left[{ }^{\prime \prime}\right]} \\
(3) \\
\end{array}$} & \multirow{3}{*}{$\begin{array}{c}\delta_{\text {opt }}-\delta_{\text {dyn }} \\
{\left[{ }^{\prime \prime}\right]} \\
(3)\end{array}$} \\
\hline & $\alpha(\mathrm{J} 2000.0)$ & $\delta(\mathrm{J} 2000.0)$ & & & & & & \\
\hline & (2) & (2) & & & & & & \\
\hline NGC 2366 & $07^{\mathrm{h}} 28^{\mathrm{m}} 53.6^{\mathrm{s}}$ & $+69^{\circ} 12^{\prime} 28^{\prime \prime}$ & 98 & 50 & 63 & 43 & -5 & -29 \\
\hline ESO 059-G001 & $07^{\mathrm{h}} 31^{\mathrm{m}} 18.0^{\mathrm{s}}$ & $-68^{\circ} 11^{\prime} 13^{\prime \prime}$ & 528 & 69 & 44 & 325 & -1 & +2 \\
\hline ESO 215-G?009 & $10^{\mathrm{h}} 57^{\mathrm{m}} 33.0^{\mathrm{s}}$ & $-48^{\circ} 10^{\prime} 47^{\prime \prime}$ & 599 & 77 & 28 & 119 & +31 & -4 \\
\hline NGC 4861 & $12^{\mathrm{h}} 59^{\mathrm{m}} 01.4^{\mathrm{s}}$ & $+34^{\circ} 51^{\prime} 44^{\prime \prime}$ & 835 & 46 & 67 & 15 & -11 & +10 \\
\hline NGC 5408 & $14^{\mathrm{h}} 03^{\mathrm{m}} 22.7^{\mathrm{s}}$ & $-41^{\circ} 22^{\prime} 12^{\prime \prime}$ & 502 & 53 & 58 & 300 & +20 & +28 \\
\hline IC 5152 & $22^{\mathrm{h}} 02^{\mathrm{m}} 41.9^{\mathrm{s}}$ & $-51^{\circ} 17^{\prime} 45^{\prime \prime}$ & 123 & 44 & 52 & 284 & +4 & +2 \\
\hline
\end{tabular}

Notes: (1) The name of the galaxy; (2) kinematic parameters derived by fitting a tilted-ring model to the H I Hermite velocity fields, the position angle is measured counter-clockwise from north to the receding side of the galaxies; (3) position offset of the dynamical centre in comparison to the optical centre.

mean velocities are biased towards the longest tail of the velocity profiles as soon as the distribution is not symmetric (see also discussion in $\mathrm{dB} 08$ ). In order to separate true emission from noise, we set some limits for the fitting algorithm (see dB08): the fitted profiles need to have amplitudes higher than $3 \sigma_{\text {chan }}$, where $\sigma_{\text {chan }}$ is the average noise in the line-free velocity channels of the cube. The minimum dispersion to be fitted has to be higher than the spectral resolution of the cube (see Table 2 for the noise values and channel separations). After creating the velocity maps, a small amount of noise pixels has to be removed that was admitted by the filter criteria. Therefore, we again follow dB08 and use the integrated $\mathrm{H}$ I column density map as a mask: fits are only retained if the total flux in the integrated H I map is higher than $3 \sigma_{\mathrm{N}}$ where $\sigma_{\mathrm{N}}$ is the noise in the integrated H I map. It is defined as $\sqrt{N} \sigma_{\text {chan }}$, where $N$ is the number of channels with signal contributing to each pixel. No smoothing is applied. Altogether, this gives us high quality velocity fields.

\section{The velocity fields and the rotation curves}

Figure 1 shows the H I velocity fields (left panels). In order to derive a rotation curve, we use the GIPSY task rotcur: tilted rings of half the beam width are fitted to both sides of the H I velocity fields. The systemic velocity, the centre position, the inclination, and the position angle of each ring are iteratively defined by keeping all parameters fixed except for the one we want to measure. As initial estimates we use kinematic parameters derived from fitting isophotes to the integrated H I intensity map (GIPSY task ellfit). The final rotation curve is then created by keeping the best-fitting parameters fixed. We also derive rotation curves for the receding and approaching side only by keeping the systemic velocity and the centre position fixed to the values obtained in the joint approach and by defining the inclination and the position angle iteratively.

The resulting rotation curves of all sample galaxies are shown in the middle panels of Fig. 1. The error bars of the rotation curves indicate the rotation curves of the receding (top) and approaching side (bottom). We then take the iteratively defined kinematic parameters from the tilted-ring analysis (see Table 3) in order to create a model velocity field for each galaxy, which is subsequently subtracted from the observed velocity field. This shows how well the kinematics of each galaxy have been measured in the tilted-ring analysis. The residual maps are presented in Fig. 1, right panels. In most of the cases, the derived parameters describe the kinematics of the galaxies quite well and the absolute values of the residuals are below an absolute value of $10 \mathrm{~km} \mathrm{~s}^{-1}$. A comparison of the positions of the optical and dynamic centres shows that the offsets (given in the last two columns of Table 3) are generally smaller than one beam size.

A different test to prove the quality of the rotation curves was done, e.g., in vE09a for NGC 2366. They chose different approaches in order to derive the rotation curve. First, the initial estimates from ellfit were kept fixed. Then, a rotation curve with the best-fitting parameters kept fixed was derived (see above). As a third approach, the best-fitting parameters were left free. vE09a could show that the deviations between these three approaches are generally small with higher deviations in the outer parts of the rotation curve. In the inner $1 \mathrm{kpc}$, the deviations are less than $\pm 2 \mathrm{~km} \mathrm{~s}^{-1}$. We perform this test for all galaxies of our sample and generally measure equally small deviations in velocity, at least in the inner kpc.

The neutral gas distribution in ESO 215-G?009 and its kinematics (including the derivation of a rotation curve) have been studied in detail by Warren et al. (2004). Rotation curves of ESO 059-G001 and ESO 215-G?009 have also been derived by Kirby et al. (2009). A comparison reveals that their parameters are in good agreement with our values.

\section{Harmonic decomposition}

In order to search for non-circular motions, we decompose the velocities detected along the tilted rings into multiple terms of sine and cosine. Following Schoenmakers (1999), the line of sight velocity $v_{\text {los }}$ can be described as:

$v_{\mathrm{los}}(r)=v_{\mathrm{sys}}(r)+\sum_{m=1}^{N} c_{m}(r) \cos m \psi+s_{m}(r) \sin m \psi$,

where $N$ is the maximum fit order used, $r$ is the radial distance from the dynamic centre, $\psi$ is the azimuthal angle in the plane of the disc, and $v_{\text {sys }}$ is the 0th order harmonic component $c_{0}$. As shown in CT08, a decomposition of the velocity field up to third order is sufficient to capture most of the non-circular signal. Therefore, we restrict our analysis to $N=3$. In the case of purely circular motion, only $m=0$ and $m=1$ terms are included in Eq. (1).

The decomposition is performed with the GIPSY task reswri, which fits a tilted-ring model to the original velocity field using circular rotation, and decomposes the line-of-sight velocity along each ring into multiple terms of sine and cosine. Subsequently, a model velocity field is created and subtracted from the original velocity field producing a residual velocity field. Note that the centre coordinates should be kept fixed (see Schoenmakers 1999). We run the routine with two different parameter sets: in the first case, all parameters except for the centre 

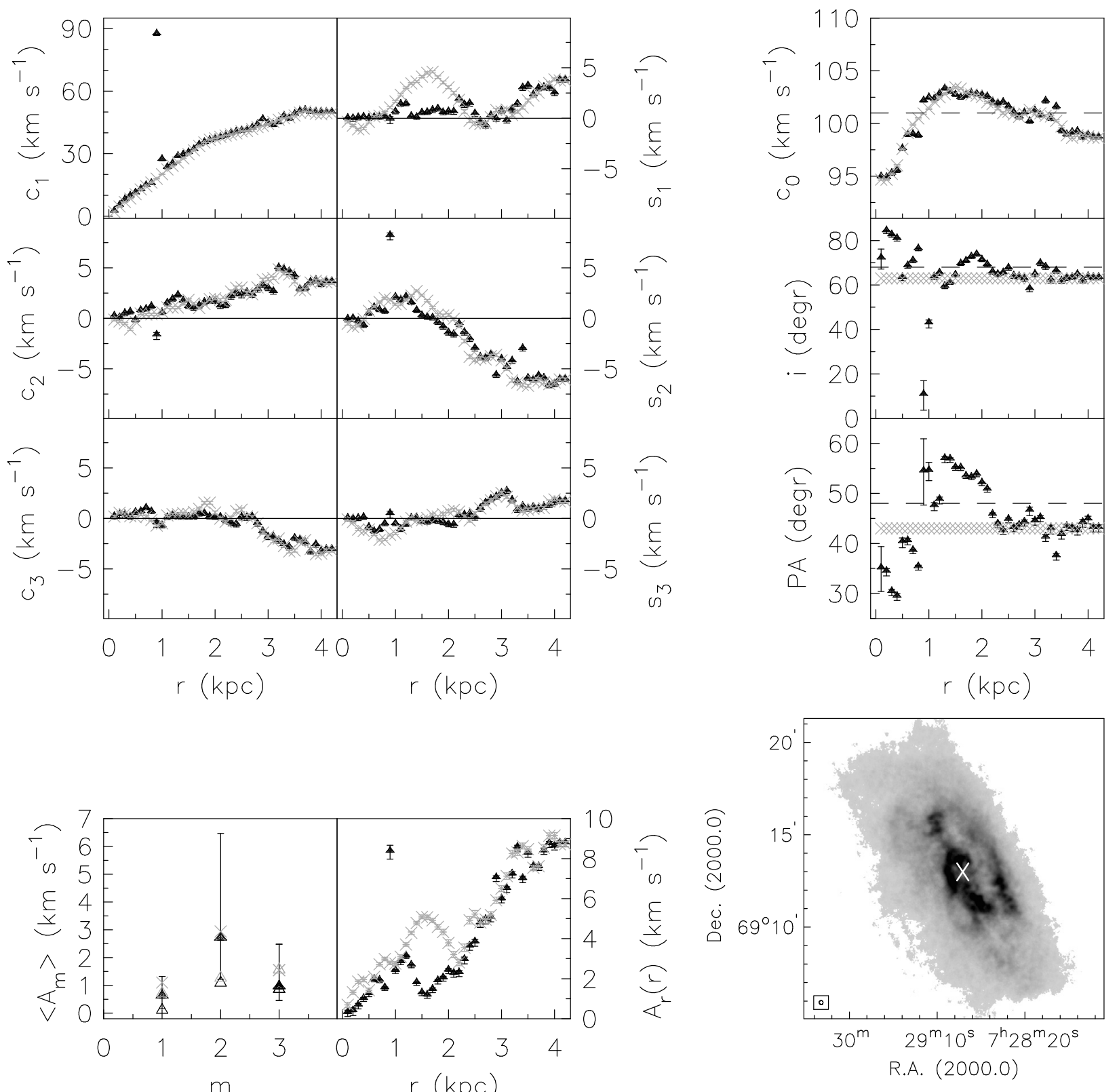

Fig. 2. The results of the unconstrained (black triangles) and constrained (light grey crosses) harmonic decomposition of NGC 2366. For the constrained fit the inclination and the position angle were fixed to the values given in Table 3. Upper left: following Eq. (1), circular $\left(c_{1}\right)$ and noncircular $\left(c_{2}, c_{3}, s_{1}, s_{2}, s_{3}\right)$ harmonic components corrected for inclination and plotted vs. radius $r$. Upper right: systemic velocity $c_{0}$, inclination $i$, and position angle $P A$, again plotted vs. radius $r$. The dashed horizontal lines represent the error weighted means of the unconstrained fit. The inclination and position angle are from the tilted-ring fit assuming circular rotation. For the unconstrained case the error bars represent the formal errors. Lower left: the median amplitudes of the individual harmonic components derived from Eqs. (2) and (3) plotted vs. harmonic number $m$. The error bars denote the upper and lower quartile of the distribution of the unconstrained $\left\langle A_{m}\right\rangle$. The open triangles represent the median amplitudes within the inner $1 \mathrm{kpc}$. Lower middle: $A_{\mathrm{r}}(r)$, the quadratically-added amplitude of all non-circular components derived from Eq. (4). The uncertainties are estimated using Gaussian error propagation. Lower right: the $\mathrm{H}$ I intensity distribution. The dynamic centre is marked by a white cross.

position (listed in Table 3) are left free. In the second case, inclination and position angle are fixed to the values given in Table 3.

The quadratically-added amplitude for each order of the harmonic decomposition is calculated using

$A_{1}(r)=\sqrt{s_{1}^{2}(r)}$

for $m=1$ ( $c_{1}$ is the circular rotation velocity) and

$A_{m}(r)=\sqrt{c_{m}^{2}(r)+s_{m}^{2}(r)}$ for $m>1$. Additionally, the quadratically-added amplitude of all non-circular harmonic components (up to $N=3$ in this case) was calculated from

$$
A_{\mathrm{r}}(r)=\sqrt{s_{1}^{2}(r)+c_{2}^{2}(r)+s_{2}^{2}(r)+c_{3}^{2}(r)+s_{3}^{2}(r)}
$$

We also derive the median value of $A_{m}(r)$ for each harmonic order $m$ in two different ways: once, using the entire radial range and once, using the inner $1 \mathrm{kpc}$ only, which is the region where the distinction between a cusp and a core becomes most obvious 

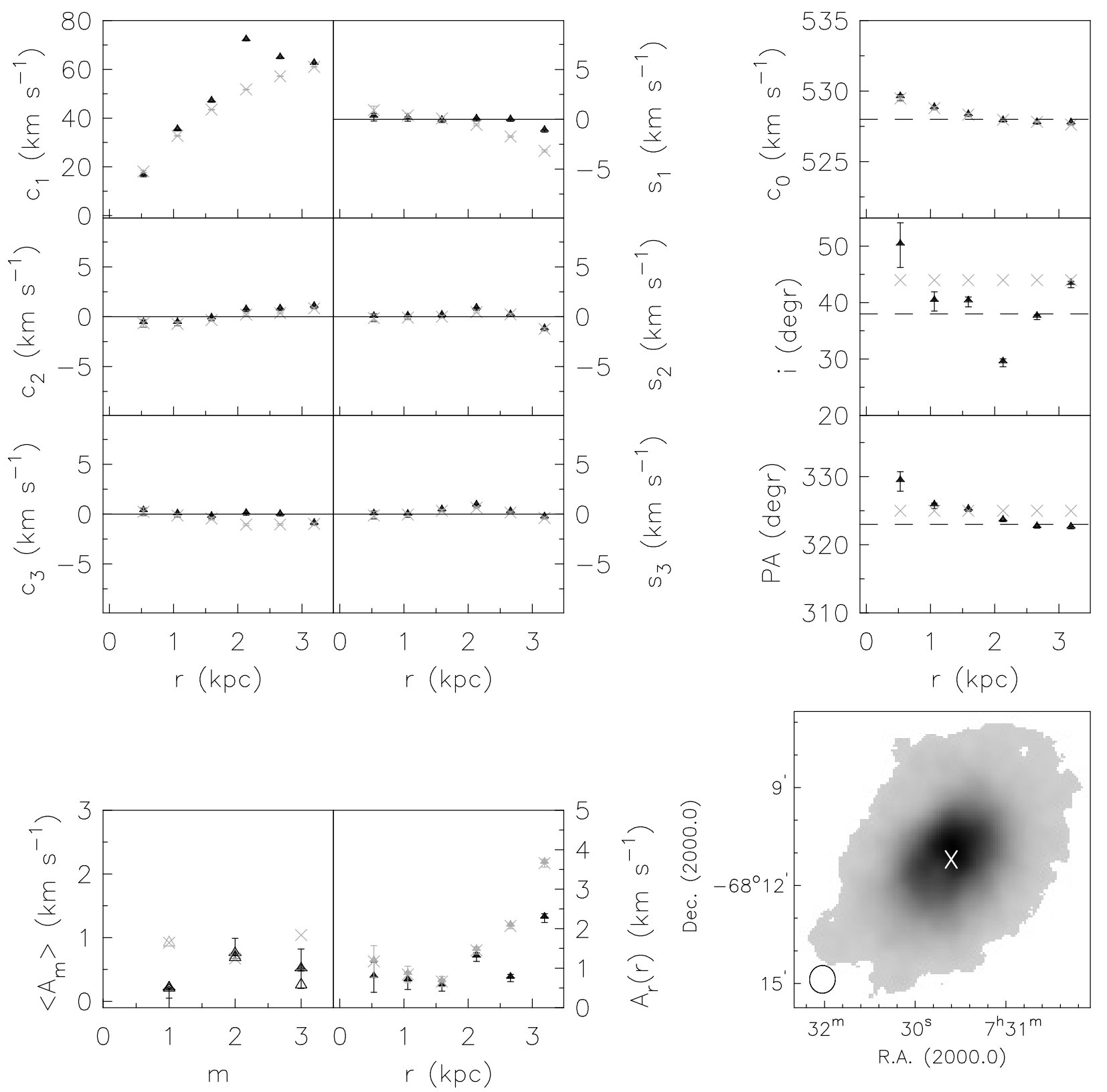

Fig. 3. The same as in Fig. 2 for ESO 059-G001. The inclination and the position angle for the constrained fit are listed in Table 3.

(de Blok 2004). More information about harmonic decompositions in general can be found in Schoenmakers et al. (1997).

In the following subsections the results for each galaxy are discussed separately. For a better understanding of the interpretation, see the rules of thumb given in Schoenmakers (1999).

\subsection{NGC 2366}

Using accurately defined centre coordinates, CT08 performed a harmonic decomposition of the Hermite velocity field of NGC 2366 (see also dB08). We also use the Hermite velocity field, but different centre coordinates (see Table 3). The radial distribution of all fitted parameters is shown in Fig. 2. The black filled triangles represent the case where all parameters except for the centre position are left free. The inclination varies significantly in the inner $1.5 \mathrm{kpc}$. As NGC 2366 is dominated by close to solid body rotation, it is difficult to determine $v_{\text {rot }}$ and $i$ simultaneously. At a radius of $1 \mathrm{kpc}, c_{0}$ rises from 95 to $102 \mathrm{~km} \mathrm{~s}^{-1}$ and the position angle jumps from about $35^{\circ}$ to $55^{\circ}$. At the same radius, the inclination has very low values, which leads to very high values of the inclination-corrected $c_{1}$. This is probably caused by the sudden decrease in H I intensity. At a radius of about $3 \mathrm{kpc}$, the $s_{1}$ and $s_{3}$ terms show the characteristic "wiggles" caused by spiral arms. As discussed by Tikhonov \& Galazutdinova (2008) and vE09a, NGC 2366 shows evidence for two weak spiral arms.

The light grey crosses in Fig. 2 show the constrained case where we use a fixed inclination of $63^{\circ}$ and a fixed position angle of $43^{\circ}$ (see Table 3 ). For most of the tilted rings, the values are in very good agreement with the ones of the unconstrained fit. A pronounced deviation can be seen in the $s_{1}$ term, where the constrained fit leads to higher amplitudes between 1 and $2 \mathrm{kpc}$ than the unconstrained fit. This is due to the constant position angle which differs significantly from the unconstrained values. 
Table 4. Derived quantities from the harmonic decomposition.

\begin{tabular}{|c|c|c|c|c|c|c|c|c|c|}
\hline $\begin{array}{l}\text { Galaxy } \\
\text { (1) } \\
\end{array}$ & $\begin{array}{c}\left\langle A_{\mathrm{r}}\right\rangle \\
{\left[\mathrm{km} \mathrm{s}^{-1}\right]} \\
(2)\end{array}$ & $\begin{array}{c}\left\langle A_{\mathrm{r}, 1 \mathrm{kpc}}\right\rangle \\
{\left[\mathrm{km} \mathrm{s}^{-1}\right]} \\
(3)\end{array}$ & $\begin{array}{c}\left\langle A_{\mathrm{r}}\right\rangle / v_{\mathrm{rot}} \\
{[\%]} \\
(4)\end{array}$ & $\begin{array}{c}\left\langle M_{\mathrm{res}}\right\rangle \\
{\left[\mathrm{km} \mathrm{s}^{-1}\right]} \\
(5)\end{array}$ & $\begin{array}{c}\left\langle A_{\mathrm{r}, \mathrm{c}}\right\rangle \\
{\left[\mathrm{km} \mathrm{s}^{-1}\right]} \\
(6)\end{array}$ & $\begin{array}{c}\left\langle A_{\mathrm{r}, \mathrm{c}, 1 \mathrm{kpc}}\right\rangle \\
{[\%]} \\
(7)\end{array}$ & $\begin{array}{c}\left\langle A_{\mathrm{r}, \mathrm{c}}\right\rangle / v_{\mathrm{rot}} \\
{\left[\mathrm{km} \mathrm{s}^{-1}\right]} \\
(8)\end{array}$ & $\begin{array}{c}\left\langle M_{\text {res, },}\right\rangle \\
{[\mathrm{kpc}]} \\
(9) \\
\end{array}$ & $\begin{array}{l}r_{\text {max }} \\
\text { (10) }\end{array}$ \\
\hline NGC 2366 & $2.91_{-1.06}^{+4.09}$ & $1.50_{-0.87}^{+0.42}$ & 5.8 & 2.15 & $4.81_{-1.83}^{+2.32}$ & $2.08_{-0.67}^{+0.66}$ & 9.6 & 1.89 & 4.1 \\
\hline ESO 059-G001 & $0.77_{-0.09}^{+0.51}$ & $0.77 \ldots$ & 1.1 & 0.43 & $1.45_{-0.61}^{+0.62}$ & $1.17 \ldots$ & 2.1 & 0.37 & 3.2 \\
\hline ESO 215-G?009 & $\ldots$ & $\ldots$ & $\ldots$ & $\ldots$ & $6.57_{-1.38}^{+1.64}$ & $5.42_{-3.03}^{+2.79}$ & 8.5 & 1.99 & 7.3 \\
\hline NGC 4861 & $1.54_{-0.46}^{+0.94}$ & $0.78 \ldots$ & 3.3 & 1.04 & $2.60_{-0.28}^{+0.78}$ & $1.02 \ldots$ & 5.7 & 1.07 & 7.1 \\
\hline NGC 5408 & $2.15_{-0.88}^{+2.62}$ & $2.50 \ldots$ & 4.1 & 1.92 & $4.08_{-1.79}^{+2.63}$ & $2.54 \ldots$ & 7.7 & 1.30 & 6.9 \\
\hline IC 5152 & $2.35_{-1.24}^{+2.27}$ & $1.63_{-1.24}^{+0.99}$ & 5.3 & 1.17 & $5.38_{-0.64}^{+0.10}$ & $5.38_{-0.64}^{+0.10}$ & 12.2 & 1.07 & 3.9 \\
\hline
\end{tabular}

Notes: (1) The name of the galaxy; (2) the median of the quadratically-added amplitude of the non-circular motions (unconstrained case), averaged over the entire radial range, the errors indicate the lower and upper quartile; (3) same as (2), but averaged over the inner $1 \mathrm{kpc}$ only; (4) the percentage the non-circular motions contribute to the maximum rotation velocity; (5) the median of the reswri absolute residual velocity field after the harmonic decomposition; (6), (7), (8), (9) the same as (2), (3), (4), (5), but for the constrained case; (10) maximum radius for the averaging of the quadratically-added amplitudes.

The median value of the quadratically-added amplitude for each order is given in the lower left panel of Fig. 2. In both cases the amplitudes are below $3 \mathrm{~km} \mathrm{~s}^{-1}$. As the innermost region of a galaxy is most significant for the cusp-core debate (de Blok 2004), we also show the median values within $1 \mathrm{kpc}$ (open triangles). These are generally below $1 \mathrm{~km} \mathrm{~s}^{-1}$ and therefore even smaller than the ones averaged over the entire radial range. $A_{\mathrm{r}}(r)$ is below $3 \mathrm{~km} \mathrm{~s}^{-1}$ in the inner $2.5 \mathrm{kpc}$ and rises to $9 \mathrm{~km} \mathrm{~s}^{-1}$ in the outer parts, contributing about $9 \%$ (constrained case) and $6 \%$ (unconstrained case) to the maximum rotation velocity of NGC 2366 (see Table 4).

In general, our results agree very well with the results of CT08 despite the different centre coordinates. This is to be expected, as CT08 have already addressed the problem of inaccurate centre positions. They showed that for dwarf galaxies, which are characterised by solid body rotation in the inner parts, the results of the harmonic decomposition are insensitive to small offsets in the centre position.

\subsection{ESO059-G001}

The results of the harmonic decomposition of ESO 059-G001 are shown in Fig. 3. The inclination at $2 \mathrm{kpc}$ drops to $30^{\circ}$, which leads to deviations in the rotation velocity. The position angle varies only slightly over the entire radial range. The amplitudes of the non-circular components are the smallest of all galaxies studied here. Parodi et al. (2002) report "rudiments of spiral arms" in ESO 059-G001, which can, however, not be seen in the harmonic components (probably due to their weakness and the relatively low spatial resolution of the $\mathrm{H} \mathrm{I}$ data).

The median amplitudes are below $1 \mathrm{~km} \mathrm{~s}^{-1}$. $A_{\mathrm{r}}(r)$ stays below $3 \mathrm{~km} \mathrm{~s}^{-1}$ over the entire radial range and only contributes $2 \%$ (constrained case) and 1\% (unconstrained case) to the maximum rotation velocity (see Table 4).

ESO 059-G001 is dominated by close to solid body rotation and additionally, the inclination is low. A tilted-ring analysis with free parameters is therefore difficult to perform. However, the constrained case with a fixed inclination of $44^{\circ}$ and a position angle of $325^{\circ}$ still gives acceptable values. As long as $i$ does not differ too much from the median value, the fits are in good agreement with the unconstrained ones. The residual map given in Fig. 1 also shows that the kinematic parameters derived from the constrained approach represent the galaxy quite well.

\subsection{ESO215-G?009}

ESO 215-G?009 has a strongly varying and very low inclination in the inner $3.5 \mathrm{kpc}$ (see Fig. 4). As shown for ESO 059-G001, a low inclination makes it very difficult to perform a tilted-ring analysis by leaving all parameters free. The partially unreasonable values for the inclination in the inner $3.5 \mathrm{kpc}$ of the unconstrained case (with $i$ as low as $5^{\circ}$ ) lead to an unphysical rotation curve. A constrained fit with fixed inclination and position angle (see Table 3) gives much more reasonable results which are, apart from the inner $3.5 \mathrm{kpc}$, largely consistent with the results from the unconstrained case. Therefore, we decide to ignore the unconstrained case for the subsequent analysis of ESO 215-G?009 and to use the constrained case instead.

Most of the amplitudes of the harmonic components are close to zero. The values of $A_{\mathrm{r}}(r)$ are typically below $10 \mathrm{~km} \mathrm{~s}^{-1}$ contributing about $8.5 \%$ to the maximum rotation velocity (see Table 4). The median amplitudes are below $5 \mathrm{~km} \mathrm{~s}^{-1}$, in the inner kpc below $3 \mathrm{~km} \mathrm{~s}^{-1}$.

We also show the results of the unconstrained fit: the low inclination causes very large circular velocity components (note that we do not plot the values between 1 and $3 \mathrm{kpc}$ that have high formal errors). The low inclination also becomes noticeable in the non-circular components, which are partly as high as $20 \mathrm{~km} \mathrm{~s}^{-1}$.

\subsection{NGC 4861}

The results of the harmonic decomposition of NGC 4861 are given in Fig. 5. The amplitudes of the non-circular components are usually close to zero. No pronounced deviation can be seen, which fits into the picture of a quiescent galaxy without spiral arm structure as described in van Eymeren et al. (2009b). The median values of the quadratically-added amplitudes for each harmonic order are below $2.5 \mathrm{~km} \mathrm{~s}^{-1}$ over the entire radial range and even below $1 \mathrm{~km} \mathrm{~s}^{-1}$ in the inner $1 \mathrm{kpc}$. The distribution of $A_{\mathrm{r}}(r)$ shows that the amplitude is generally below $3 \mathrm{~km} \mathrm{~s}^{-1}$, except for the outermost part. On average, the non-circular motions contribute about 6\% (constrained case) and 3\% (unconstrained case) to the maximum rotation velocity. The amplitudes of the harmonic components of the constrained fit (inclination of $67^{\circ}$, position angle of $15^{\circ}$ ) usually agree to within $2 \mathrm{~km} \mathrm{~s}^{-1}$ with the ones from the unconstrained fit. 

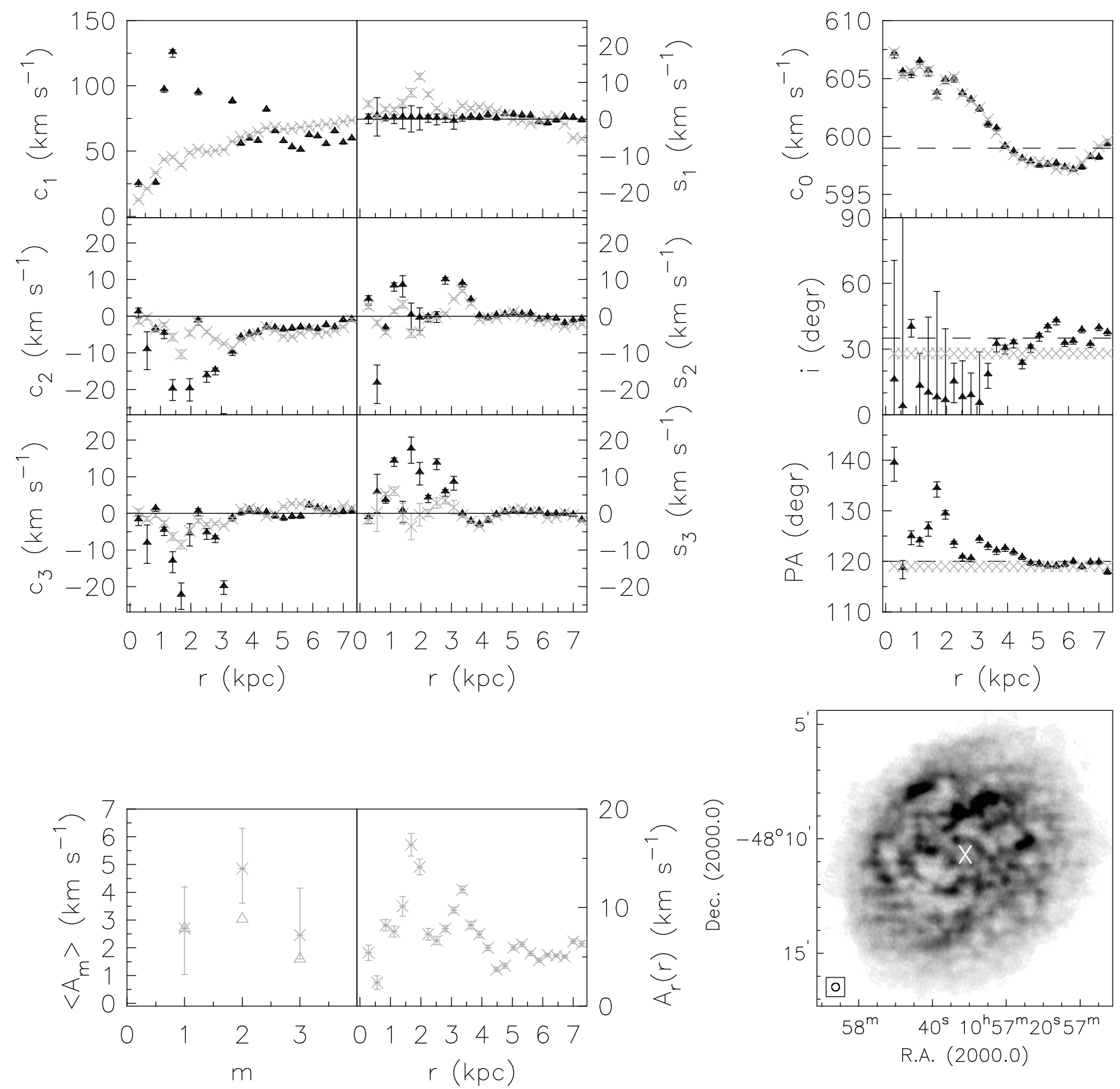

Fig. 4. The same as in Fig. 2 for ESO 215-G?009. The inclination and the position angle for the constrained fit are listed in Table 3. The analysis of this galaxy is based on the constrained case (see Sect. 4.3). Therefore, no $\left\langle A_{m}\right\rangle$ and $A_{\mathrm{r}}(r)$ are given for the unconstrained case.

\subsection{NGC5408}

Figure 6 shows the results for NGC 5408. In her $\mathrm{PhD}$ thesis, van Eymeren (2008) suggested that this galaxy has two kinematic systems, which is probably the reason for the clear and welldefined radial variation of the inclination in the unconstrained fit. Nevertheless, the amplitudes of the non-circular motions are small. $A_{\mathrm{r}}(r)$ is below $3 \mathrm{~km} \mathrm{~s}^{-1}$ in the inner $5 \mathrm{kpc}$ and rises to about $6 \mathrm{~km} \mathrm{~s}^{-1}$ in the outer parts. On average, the non-circular motions contribute less than $8 \%$ (constrained case) and $4 \%$ (unconstrained case) to the maximum rotation velocity. The median amplitudes of the individual harmonic orders are below $3 \mathrm{~km} \mathrm{~s}^{-1}$, independent of the radial range we look at.

For the constrained case we use a fixed inclination of $58^{\circ}$ and a fixed position angle of $300^{\circ}$ (see Table 3 ). In the inner $2.5 \mathrm{kpc}$ this inclination agrees with the one from the unconstrained fit.
However, it differs significantly from the inclination of the outer kinematic system. For $r>2.5 \mathrm{kpc}$ this results in different inclination corrections for the constrained and the unconstrained cases, which can be seen, e.g., in the small offset in $c_{1}$.

\subsection{IC 5152}

Except for one outlier in the inner $1 \mathrm{kpc}$, the inclination of IC 5152 is roughly constant with radius (see Fig. 7). The position angle increases at $2 \mathrm{kpc}$ by about $20^{\circ}$. Together, this leads to small non-circular components, often close to $0 \mathrm{~km} \mathrm{~s}^{-1}$. The constrained case (with an inclination of $45^{\circ}$ and a position angle of $287^{\circ}$ ) and the unconstrained case are in good agreement, although the fixed position angle leads to higher amplitudes in the $s_{1}$ term. $A_{\mathrm{r}}(r)$ varies over the entire radial range, but stays 

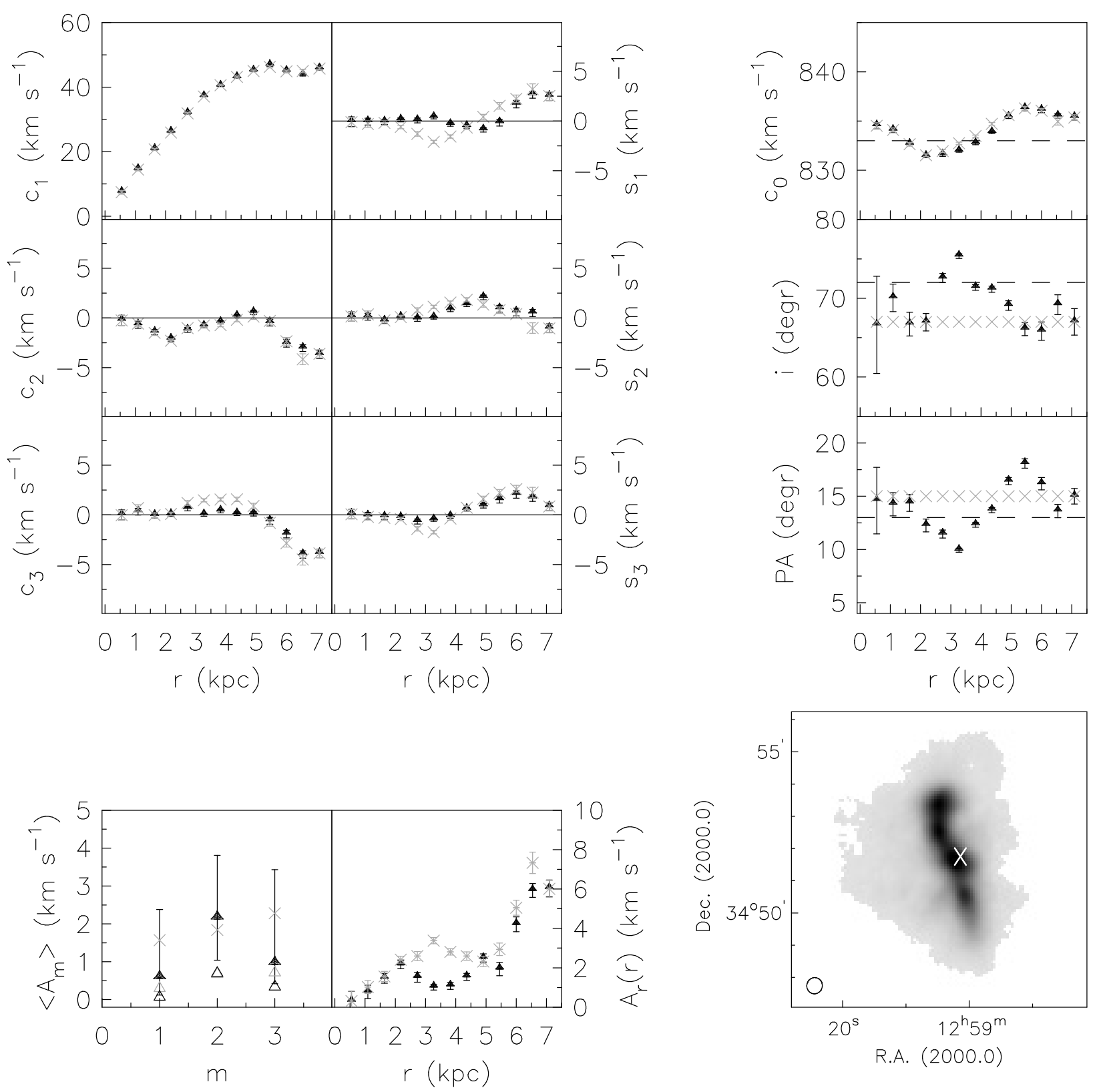

Fig. 5. The same as in Fig. 2 for NGC 4861. The inclination and the position angle for the constrained fit are listed in Table 3.

below $8 \mathrm{~km} \mathrm{~s}^{-1}$. The difference between the amplitudes of the constrained and unconstrained values are several $\mathrm{km} \mathrm{s}^{-1}$, which is due to the difference in the $s_{1}$ term. The median amplitudes of the individual harmonic orders are below $2 \mathrm{~km} \mathrm{~s}^{-1}$ for the unconstrained case. In order to calculate the median amplitudes in the inner $1 \mathrm{kpc}$, we removed the outlier at $0.5 \mathrm{kpc}$. The $m=1$ term of the constrained case is especially high, which is again due to the high amplitudes of the $s_{1}$ term. In comparison to the other galaxies of our sample, IC 5152 shows the highest contribution of non-circular motions to the maximum rotation velocity.

\subsection{Discussion}

In all sample galaxies, the non-circular motions contribute on average about $8 \%$ (constrained case) and about $4 \%$ (unconstrained case) to the maximum rotation velocity. However, in the inner few kpcs the rotation velocity has not yet reached its maximum value. Furthermore, for the cusp-core debate the inner few kpcs are most relevant, whereas non-circular motions in the outer parts of a galaxy, though interesting, are not relevant. Therefore, we perform a more detailed analysis of the quadratically-added amplitude of all non-circular motions within the inner $5 \mathrm{kpc}$. We concentrate on the results of the constrained case. Especially in the inner few kpcs, a strong change in inclination or position angle is not to be expected, so that it is a reasonable assumption to keep both parameters fixed. As Table 4 shows, the amplitudes of the constrained case are usually up to a factor of two higher than the amplitudes of the unconstrained case. Therefore, the constrained case gives an upper limit to the amount of non-circular motions.

Figure 8 shows the amplitudes of the constrained case averaged within rings of $1 \mathrm{kpc}$ width (left panels) and within rings of increasing radius (right panels). Furthermore, we differentiate between the absolute amplitudes (upper panels) and 

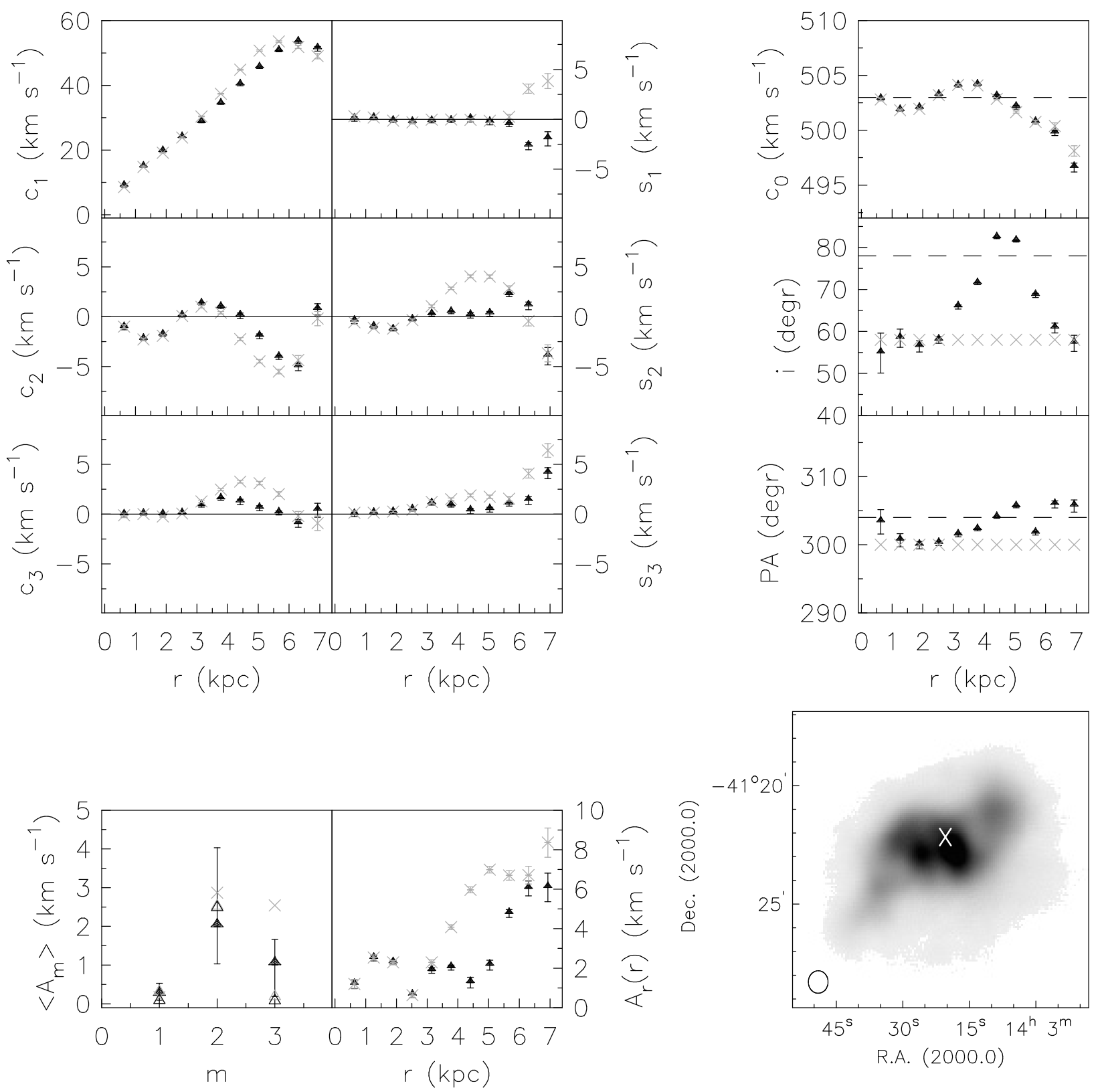

Fig. 6. The same as in Fig. 2 for NGC 5408. The inclination and the position angle for the constrained fit are listed in Table 3.

the amplitudes that are normalised by the local rotation velocity (lower panels). In all sample galaxies and for all radii, the non-circular motions generally contribute less than $25 \%$ to the local rotation velocity, sometimes even less than $10 \%$. In the inner $1 \mathrm{kpc},\left\langle A_{\mathrm{r}}(r)\right\rangle$ is often close to 1 or $2 \mathrm{~km} \mathrm{~s}^{-1}$ (see Table 4), which corresponds to about $5 \%$ to $25 \%$ of the local rotation velocity. According to the simulations by Hayashi et al. (2004) and Hayashi \& Navarro (2006), the non-circular motions add up to about $50 \%$ of the local rotation velocity at a radius of $1 \mathrm{kpc}$ and even more below $1 \mathrm{kpc}$.

In order to check if our harmonic decomposition is able to quantify most of the non-circular motions, we follow CT08 and make use of the residual velocity fields. In Fig. 9 we compare the median of the absolute residual velocity fields created with reswri (constrained case) with the median of the absolute residual velocity fields created with rotcur. The galaxies from our sample are represented by open black triangles. For a comparison we also show the data points from CT08 (filled light grey triangles). In agreement with CT08, the rotcur residuals are larger than the reswri ones as rotcur does not take non-circular motions into account. In comparison to most of the galaxies studied by CT08, our sample dwarf galaxies lie at the lower end of the distribution. The median value of the reswri residuals $\left\langle M_{\text {res }}\right\rangle$ is below $2 \mathrm{~km} \mathrm{~s}^{-1}$ (see Table 4), which implies that the harmonic decomposition up to third order has captured most of the non-circular motions.

Altogether, we could show that while large non-circular motions might be found in dwarf galaxies, they are the exception rather than the rule. The galaxies in our sample have non-circular motions that are that small that they do not significantly affect the rotation curves. This implies that non-circular motions cannot artificially flatten the slope of the density profile and turn a cusp into a core. 

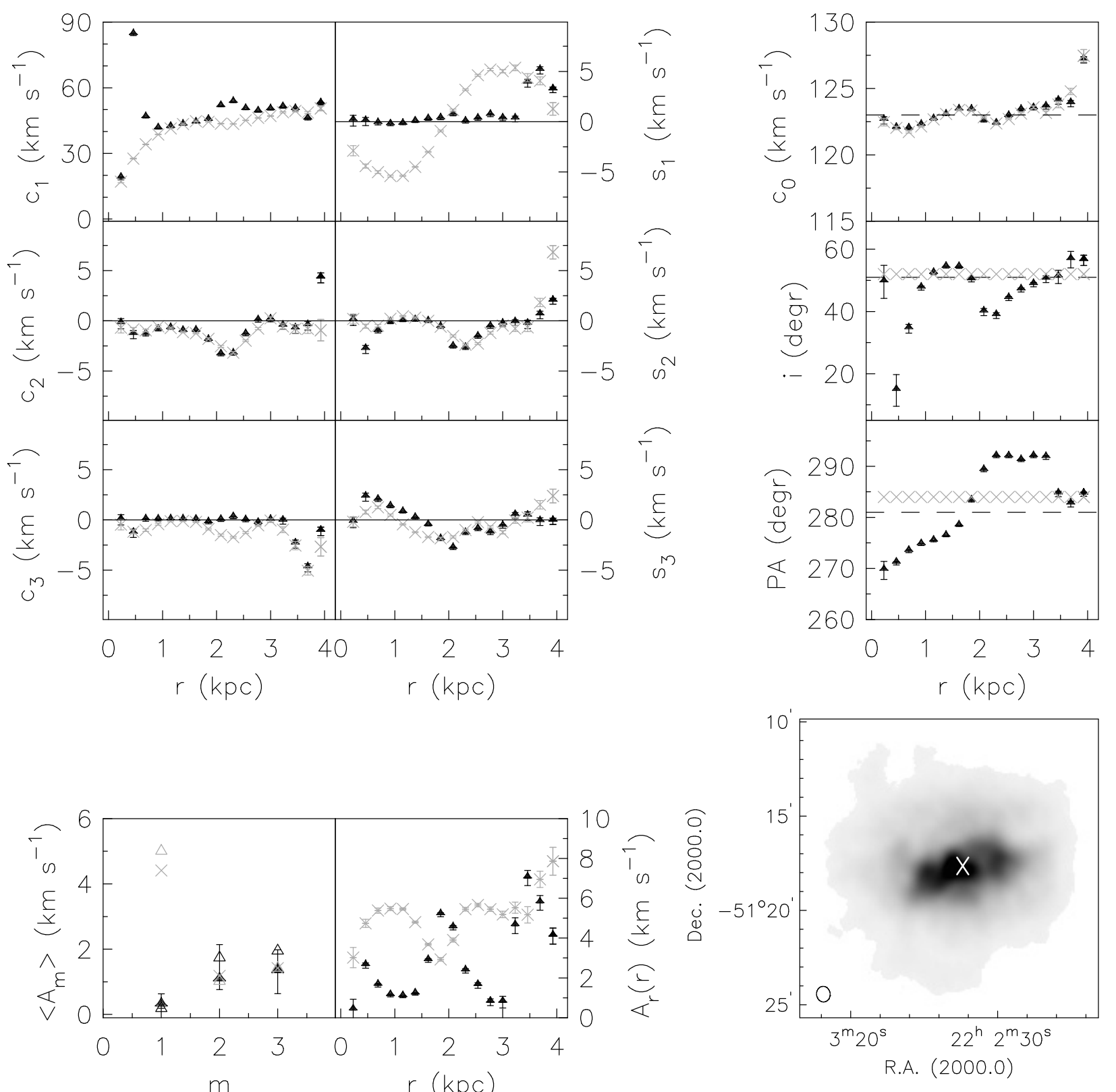

Fig. 7. The same as in Fig. 2 for IC 5152. The inclination and the position angle for the constrained fit are listed in Table 3.

\section{Mass models}

The HI rotation curves of our sample galaxies are only marginally influenced by non-circular motions. Therefore, we use the curves derived with rotcur (see Sect. 3 and Fig. 1, middle panels) to perform a mass decomposition with the GIPSY task rotmas. This task allows us to interactively fit the different components (halo, gas, stars) to the observed rotation curve by minimising the $\chi^{2}$ of the parameter space. In this section, the dark matter halo models are introduced and the derivation of the mass components is described.

\subsection{Dark matter halo models}

We use two models to describe the dark matter halo, which are the cuspy NFW halo (e.g., Navarro et al. 1996) and the cored pseudo-isothermal (ISO) halo (e.g., Binney \& Tremaine 1987).
Numerical simulations show that the density of CDM haloes rises steeply towards the halo centre (e.g., Navarro et al. 1996). The NFW mass-density distribution is described as

$\rho_{\mathrm{NFW}}(r)=\frac{\rho_{i}}{r / r_{\mathrm{s}} \cdot\left(1+r / r_{\mathrm{s}}\right)^{2}}$,

where $\rho_{i}$ is related to the density of the Universe at the time of halo collapse, and $r_{\mathrm{s}}$ is the characteristic radius of the halo. This leads to a rotation curve of

$v_{\text {rot }}(r)=v_{200} \sqrt{\frac{\ln (1+c x)-(c x) /(1+c x)}{x[\ln (1+c)-c /(1+c)]}}$,

with $x=r / r_{200}$. The concentration parameter $c=r_{200} / r_{\mathrm{s}}$ is directly correlated to $v_{200}$, the circular velocity at $r_{200}$ (de Blok et al. 2003)

$\log c=1.191-0.064 \log v_{200}-0.032 \log v_{200}^{2}$, 


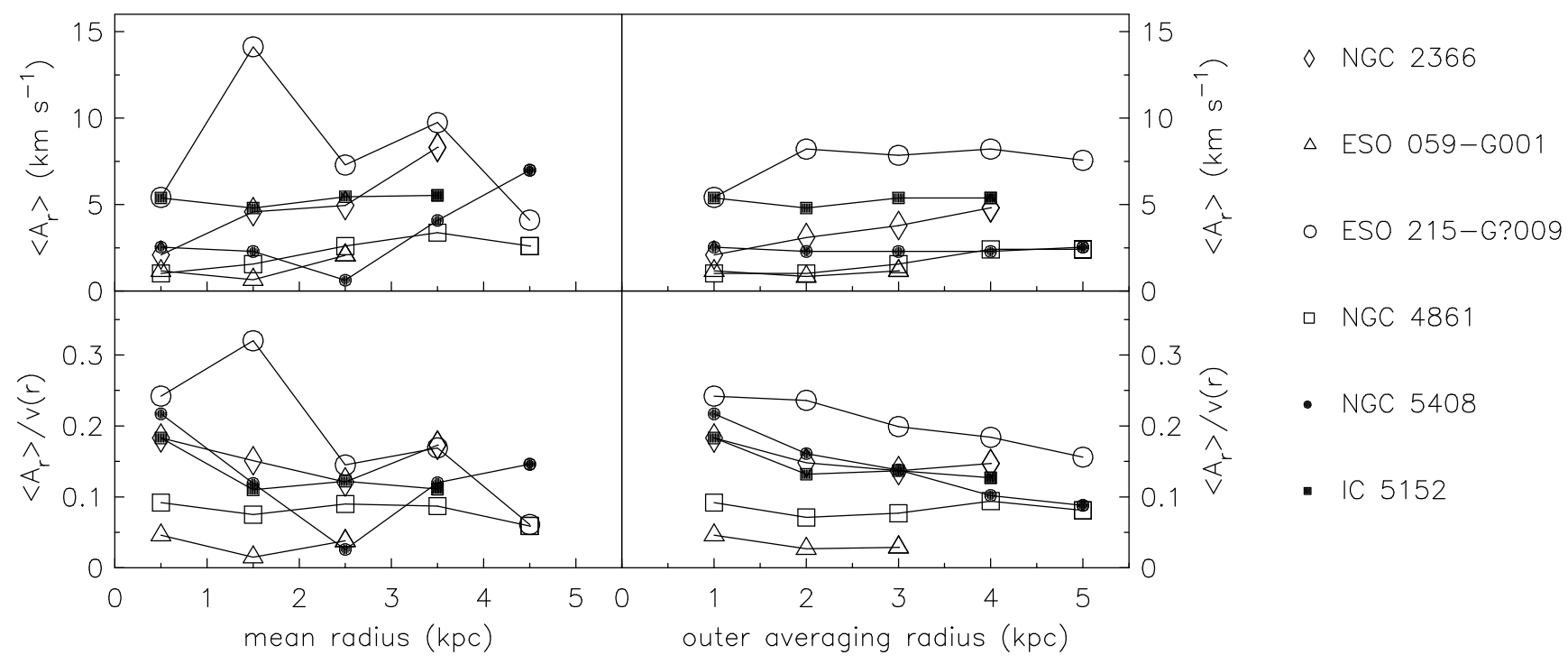

Fig. 8. The mean of the quadratically-added amplitudes derived from the constrained case. Upper left panel: the amplitudes of the non-circular motions within rings of $1 \mathrm{kpc}$ width (i.e., $0<r<1 \mathrm{kpc}, 1<r<2 \mathrm{kpc}, \ldots, 4<r<5 \mathrm{kpc}$ ) for each galaxy (indicated by different symbols). Upper right panel: the same as the upper left panel, but the amplitudes of the non-circular motions are averaged within rings of increasing radius (i.e., $0<r<1 \mathrm{kpc}, 0<r<2 \mathrm{kpc}, \ldots, 0<r<5 \mathrm{kpc}$ ). Lower left and right panel: like the upper left and right panel, but the amplitudes of the non-circular motions are normalised by the local rotation velocity.

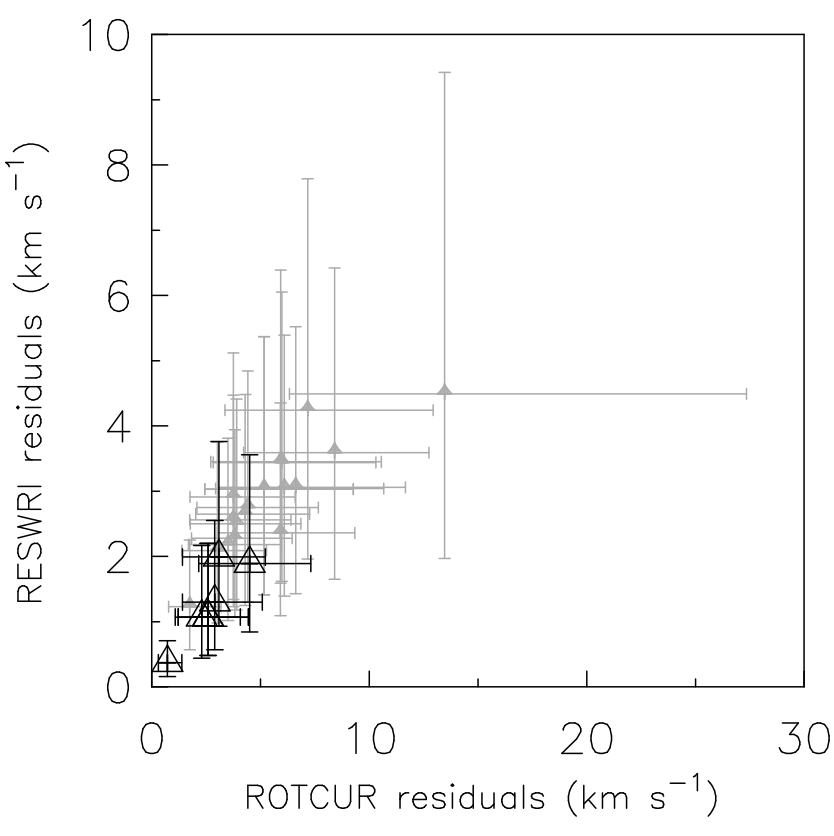

Fig. 9. Median of the absolute residual field from the harmonic decomposition (constrained case) vs. the one from the rotation curve analysis. The light grey triangles represent the values derived by CT08. They are complemented by the results of our analysis (black open triangles). The error bars indicate the lower and upper quartile.

where $r_{200}$ is the radius at which the density contrast exceeds 200, i.e., roughly the virial radius (Navarro et al. 1996). Note that we do not correct the NFW fits for adiabatic compression. As Sellwood \& McGaugh (2005) show, the central halo concentration of dwarf galaxies increases only slightly from the primordial to the compressed halo, which means that it still fits the result from $N$-body simulations $(\alpha=1)$.

As a second model the empirically derived ISO halo is used (Binney \& Tremaine 1987). It describes a dark matter halo that has a core of roughly constant density. The density profile is given by

$\rho_{\mathrm{ISO}}(r)=\rho_{0}\left(1+\left(\frac{r}{r_{\mathrm{c}}}\right)^{2}\right)^{-1}$,

with $\rho_{0}$ being the central density and $r_{\mathrm{c}}$ the core radius. The rotation curve corresponding to this density profile can be expressed as

$v_{\mathrm{rot}}(r)=\sqrt{4 \pi G \rho_{0} r_{\mathrm{c}}^{2}\left(1+\frac{r_{\mathrm{c}}}{r} \arctan \left(\frac{r}{r_{\mathrm{c}}}\right)\right)}$.

\subsection{Fitting process}

As dwarf galaxies are believed to be dark matter dominated at all radii (de Blok \& McGaugh 1997), the velocity contribution from the baryons is often neglected when decomposing the rotation curves of these galaxies. But although dark matter is the dominant component in dwarf galaxies, the baryons, i.e., gas and stars, are still important, especially close to the centre. Including all components, the observed rotation velocity is then given by

$v_{\text {obs }}^{2}=v_{\text {stars }}^{2}+v_{\text {gas }}^{2}+v_{\text {halo }}^{2}$.

We model the rotation curves in various ways, partly including the baryons. The different approaches are described in the following. 
Table 5. Parameters for the exponential disc fitting.

\begin{tabular}{lcccccc}
\hline \hline Parameter [Unit] & NGC 2366 & ESO 059-G001 & ESO 215-G?009 & NGC 4861 & NGC 5408 & IC 5152 \\
& $(1)$ & $(2)$ & $(3)$ & $(4)$ & $(5)$ & $(2)$ \\
\hline Band & $V$ & $H$ & $R$ & $R$ & $J$ & $H$ \\
$\mu_{0}[\mathrm{mag} \mathrm{arcsec}$ & -2 & 23.16 & 22.95 & 18.29 & 17.65 \\
$h[\mathrm{kpc}]$ & 22.82 & 20.23 & 2.16 & 0.88 & 0.33 & 0.36 \\
$n$ & 1.59 & 0.81 & 1 & 1 & 1 & 0.96 \\
\hline$M / L$ & 1 & 1.33 & 2 & 0.3 & $<0.1$ & 0.2 \\
\hline
\end{tabular}

References: Photometry by (1) Hunter et al. (2001); (2) Kirby et al. (2008); (3) Warren et al. (2006); (4) Gil de Paz \& Madore (2005); (5) Noeske et al. (2003).

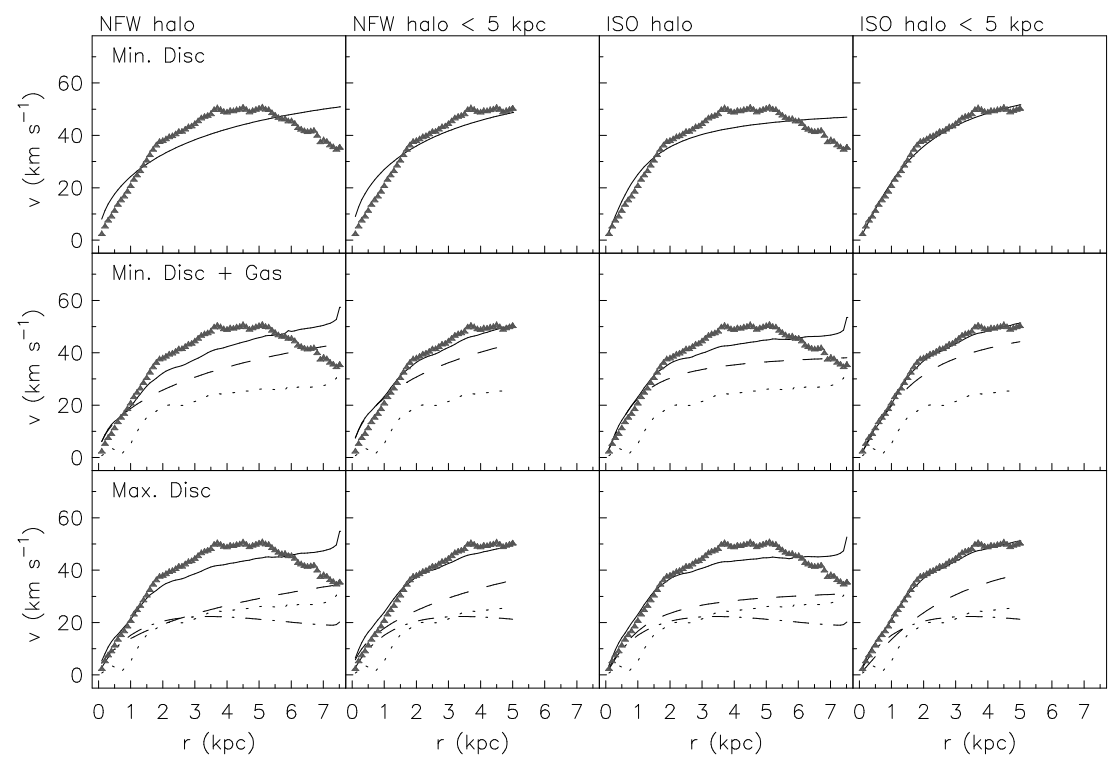

Fig. 10. Mass decomposition of NGC 2366. The rotation curves for an NFW halo and a pseudo-isothermal halo are modelled for the full rotation curve, and for the inner $5 \mathrm{kpc}$ only. From top to bottom the minimum-disc, the minimum-disc + gas, and the maximumdisc cases are fitted. Solid triangles represent the observed rotation curve, longdashed lines the dark matter halo component, dotted lines the gas component, dashed-dotted lines the contribution of the stars, and solid lines the resulting model fit.

\subsubsection{Minimum-disc case}

This is the simplest case ignoring all baryonic contributions. Thus, only the dark matter haloes are fitted to the observed rotation curve.

\subsubsection{Minimum-disc + gas case}

In this case, the contribution of the gas is taken into account as well. In addition to $\mathrm{H}$ I, which dominates the gas component, He and metals are included by scaling the H I column density by a factor of 1.4. As the ratio between the molecular gas and $\mathrm{HI}$ is much lower in dwarf galaxies than in luminous spirals (Taylor et al. 1998; Leroy et al. 2005), we do not correct for this gas component. In order to create a surface density profile for the H I, the GIPSY task ellint is taken, using the H I intensity maps (Figs. 2-7, lower right panels) and the parameters from the best tilted-ring model (see Table 3 ) as an input. The output of ellint is given in terms of mean flux and has therefore to be converted to physical units. We then use the GIPSY task rotmod in order to determine the rotation of the gas under the assumption of an infinitesimally thin disc.

\subsubsection{Maximum-disc case}

Here, the contribution of the gas, the stars, and the dark matter halo are simultaneously fitted to the observed rotation curve.
For all galaxies, the surface density can be described by a Sérsic model (Sersic 1968)

$\mu=\mu_{0}+1.086\left(\frac{r}{h}\right)^{n}$

with $\mu_{0}$ being the central surface brightness, $h$ the disc scale length, and $n$ the shape parameter (where $n=1$ gives an exponential profile). The parameters for each galaxy are listed in Table 5. They are converted from mag $\operatorname{arcsec}^{-2}$ to units of $L_{\odot} \mathrm{pc}^{-2}$. Again, we use rotmod to determine the contribution of the stars under the assumption of an infinitesimally thin disc. The derivation of the contribution of the stellar disc is the most critical part: first, the surface photometry depends on different factors which are difficult to estimate, e.g., the extinction. Secondly, in order to derive the stellar rotation curve, the mass to light ratio $M / L$ has to be known. Note that we make the assumption of a stellar $M / L$ of 1 in the case of NGC 2366 and of 2 in the case of ESO 215-G?009, which are in good agreement with adopting a Kroupa or a Kennicutt initial mass function (Portinari et al. 2004, and references therein). For the other sample galaxies, calculations by assuming a Kroupa or Kennicutt initial mass function reveal stellar $M / L$ ratios that result in a stellar rotation curve that lies above the observed one. Therefore, we choose smaller stellar $M / L$ ratios, but always greater than 0.2 , which is still a physical value (see also Spano et al. 2008). In the case of NGC 5408, we do not fit a stellar component as the $M / L$ value has to be far below 0.1 in order to fit the observed rotation curve. 


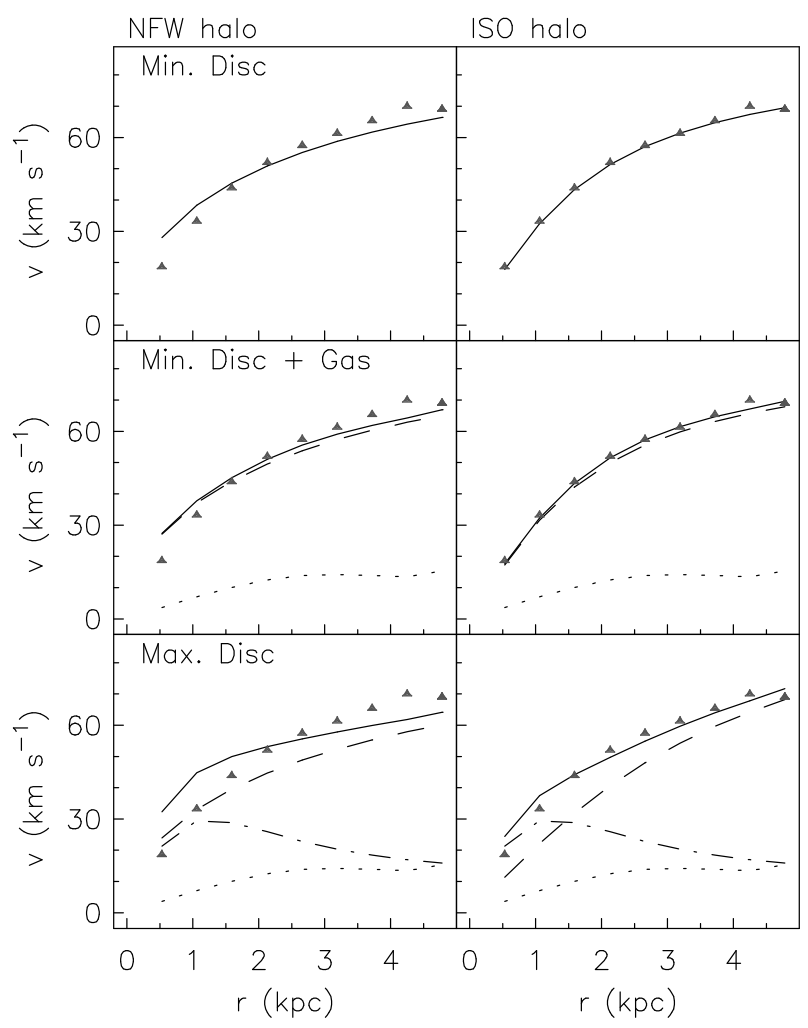

Fig. 11. Mass decomposition of ESO 059-G001. The same as in Fig. 10. No reduced rotation curve is fitted.

\section{Results and discussion}

As described in, e.g., vE09a, the outer parts of the rotation curves show large uncertainties due to the sparsely filled tilted rings. Therefore, we decompose the rotation curves twice for each of the different cases (see Sect. 5.2) and for each dark matter density profile: first, using the entire rotation curve and secondly, using a truncated rotation curve restricted to the inner few kpcs only. The results for all sample galaxies are shown in Figs. 10-15. From top to bottom the minimum-disc, the minimum-disc + gas, and the maximum-disc cases are fitted. Solid triangles represent the observed rotation curve, longdashed lines the dark matter halo component, dotted lines the gas component, and dashed-dotted lines the contribution of the stars. The resulting model fit is indicated by a solid line.

Tables 6 and 7 list the results of the best fits for both halo profiles including the reduced $\chi^{2}$ values. We assume $v_{200}$ to be the rotation speed, which we estimate from the flat parts of the observed rotation curves. Following Eq. (7), we then derive the concentration parameter $c$, which is between 9 and 10 for all sample galaxies, i.e., consistent with CDM simulations. Both parameters are kept fixed so that $r_{200}$ is the only free parameter. In the case of the ISO halo both parameters, the core radius $r_{\mathrm{c}}$ and the core density $\rho_{0}$, are allowed to vary.

In the following subsections we present the results for each galaxy separately.

\subsection{NGC 2366}

A mass decomposition of NGC 2366 has already been done by Oh08. They derived the rotation curve from a so-called "bulk" velocity field, which only contains the circularly rotating components (Oh08). Our analysis is done on the Hermite velocity field that still contains non-circular motions. However, as shown in Sect. 4.1, the non-circular motions in NGC 2366 (as well as in the other sample galaxies) do not significantly affect the rotation curves.

Figure 10 shows the resulting model fits. A comparison of the reduced $\chi^{2}$ values (see Tables 6 and 7) reveals that the ISO halo gives much better results than the NFW halo independent of the radius of the rotation curve. In the outer parts, the observed rotation curve is affected by uncertainties caused by the sparsely filled tilted rings and by asymmetries in the $\mathrm{H}$ I velocity field (cf. vE09a). Therefore, it strongly declines from a radius of $5 \mathrm{kpc}$ on so that we also model the curve within the inner $5 \mathrm{kpc}$ only. This gives improved fits for both haloes: $\chi_{\text {red }}^{2}$ is lower by a factor of four when using the NFW halo and even by a factor of 20 when using the ISO halo. Additionally, $\chi_{\text {red }}^{2}$ decreases by including gas and stars.

The reduced $\mathrm{H}$ I rotation curve fitted with an ISO halo gives the lowest values for $\chi_{\text {red }}^{2}$. The resulting model fit agrees very well with the observed curve. Moreover, the values of the core densities and radii from the ISO models are comparable to the results of $\mathrm{Oh} 08$.

\subsection{ESO 059-G001}

As the rotation velocities of this galaxy have small errors (also in the outer parts), we only perform a mass decomposition for the entire rotation curve. Figure 11 shows the resulting curves. Again, the ISO halo gives much better results than the NFW halo. Especially the models for the minimum-disc and minimum-disc + gas cases using the truncated rotation curve result in very low $\chi_{\text {red }}^{2}$ values (see Tables 6 and 7).

\subsection{ESO215-G?009}

ESO 215-G?009 is modelled over the entire radial range and over the inner $8.1 \mathrm{kpc}$ only (see Fig. 12). In both cases, the NFW halo fits sometimes even give lower $\chi_{\text {red }}^{2}$ values than the ISO halo fits. This can probably be explained by the slightly scattered rotation velocities between 1 and $3 \mathrm{kpc}$.

\subsection{NGC 4861}

Figure 13 shows the results for NGC 4861. The reduced $\chi^{2}$ values of the maximum-disc case reveal that the stars are not well represented by our estimate as the $\chi_{\text {red }}^{2}$ values increase, independent of the model, after adding the stellar component (see Tables 6 and 7). Similar to the other galaxies in our sample, the ISO matches the observed rotation curve better than the NFW halo.

\subsection{NGC 5408}

The H I rotation curve of NGC 5408 rises very slowly in the inner $2 \mathrm{kpc}$. Using the photometric data from Noeske et al. (2003) (see Table 5), it is impossible to derive a stellar rotation curve that lies below the total rotation curve, even when assuming a stellar $M / L$ ratio of 0.1 , which is the lowest value before becoming unphysical (Spano et al. 2008). We either underestimate the rotation in the central part of the galaxy due to the low spatial resolution or the photometric data are not accurate enough. We therefore decide against modelling the stellar component. 

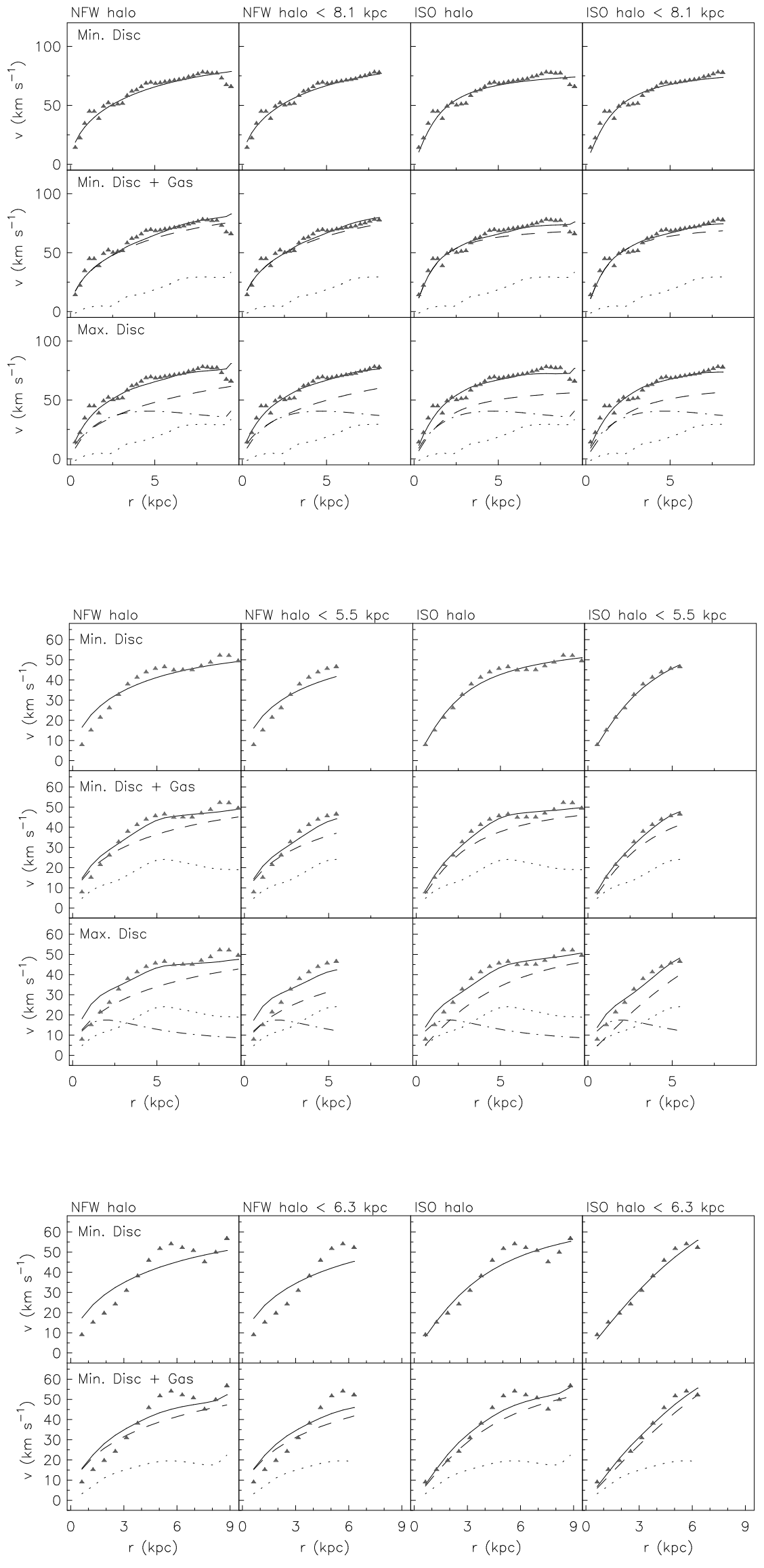

Fig. 12. Mass decomposition of ESO 215G?009. The same as in Fig. 10. A reduced rotation curve is fitted up to a radius of $8.1 \mathrm{kpc}$.

Fig. 13. Mass decomposition of NGC 4861. The same as in Fig. 10. A reduced rotation curve is fitted up to a radius of $5.5 \mathrm{kpc}$.

Fig. 14. Mass decomposition of NGC 5408. The stellar contribution cannot be fitted. A reduced rotation curve is fitted up to a radius of $6.3 \mathrm{kpc}$. Otherwise the same as in Fig. 10. 
Table 6. The basic parameters of the mass decomposition - the NFW model.

\begin{tabular}{|c|c|c|c|c|c|c|c|c|c|c|}
\hline \multirow[b]{2}{*}{$\begin{array}{l}\text { Parameter } \\
\text { [Unit] }\end{array}$} & \multicolumn{5}{|c|}{ NFW full } & \multicolumn{5}{|c|}{ NFW small } \\
\hline & $\begin{array}{c}v_{200} \\
{\left[\mathrm{~km} \mathrm{~s}^{-1}\right]} \\
(1)\end{array}$ & $\begin{array}{l}c \\
(2) \\
\end{array}$ & $\begin{array}{c}r_{200} \\
{[\mathrm{kpc}]} \\
(3)\end{array}$ & $\begin{array}{c}\Delta r_{200} \\
{[\mathrm{kpc}]} \\
(4)\end{array}$ & $\begin{array}{l}\chi_{\text {red }}^{2} \\
(5) \\
\end{array}$ & $\begin{array}{c}v_{200} \\
{\left[\mathrm{~km} \mathrm{~s}^{-1}\right]} \\
(6)\end{array}$ & $\begin{array}{l}c \\
(7) \\
\end{array}$ & $\begin{array}{c}r_{200} \\
{[\mathrm{kpc}]} \\
(8) \\
\end{array}$ & $\begin{array}{c}\Delta r_{200} \\
{[\mathrm{kpc}]} \\
(9) \\
\end{array}$ & $\begin{array}{l}\chi_{\text {red }}^{2} \\
(10) \\
\end{array}$ \\
\hline \multicolumn{11}{|c|}{ NGC 2366} \\
\hline Min.-disc & 50 & 9.4 & 117.54 & 7.26 & 47.47 & 50 & 9.4 & 92.81 & 4.52 & 19.88 \\
\hline Min.-disc + gas & 50 & 9.4 & 206.12 & 16.44 & 51.76 & 50 & 9.4 & 136.75 & 5.06 & 8.19 \\
\hline Max.-disc & 50 & 9.4 & 386.29 & 37.63 & 36.27 & 50 & 9.4 & 229.04 & 11.39 & 7.81 \\
\hline \multicolumn{11}{|c|}{ ESO 059-G001 } \\
\hline Min.-disc & 69 & 9.2 & 89.22 & 7.84 & 22.81 & $\ldots$ & $\ldots$ & $\ldots$ & $\ldots$ & $\ldots$ \\
\hline Min.-disc + gas & 69 & 9.2 & 96.00 & 8.04 & 19.67 & $\ldots$ & $\ldots$ & $\ldots$ & $\ldots$ & $\ldots$ \\
\hline Max.-disc & 69 & 9.2 & 124.40 & 21.27 & 63.70 & $\ldots$ & $\ldots$ & $\ldots$ & $\ldots$ & $\ldots$ \\
\hline \multicolumn{11}{|c|}{ ESO 215-G?009 } \\
\hline Min.-disc & 77 & 9.0 & 134.57 & 5.13 & 19.07 & 77 & 9.0 & 128.08 & 3.63 & 9.14 \\
\hline Min.-disc + gas & 77 & 9.0 & 157.35 & 7.85 & 30.18 & 77 & 9.0 & 145.41 & 5.00 & 12.48 \\
\hline Max.-disc & 77 & 9.0 & 309.86 & 17.19 & 19.95 & 77 & 9.0 & 283.81 & 11.67 & 9.13 \\
\hline \multicolumn{11}{|c|}{ NGC 4861} \\
\hline Min.-disc & 46 & 9.5 & 125.79 & 10.55 & 17.42 & 46 & 9.5 & 132.55 & 18.92 & 29.44 \\
\hline Min.-disc + gas & 46 & 9.5 & 181.48 & 13.27 & 10.93 & 46 & 9.5 & 189.54 & 24.43 & 16.57 \\
\hline Max.-disc & 46 & 9.5 & 219.91 & 26.36 & 24.86 & 46 & 9.5 & 267.28 & 65.64 & 37.31 \\
\hline \multicolumn{11}{|c|}{ NGC 5408} \\
\hline Min.-disc & 53 & 9.7 & 182.70 & 24.22 & 47.97 & 53 & 9.7 & 187.81 & 34.65 & 63.22 \\
\hline Min.-disc + gas & 53 & 9.7 & 233.18 & 30.95 & 38.76 & 53 & 9.7 & 239.76 & 46.08 & 51.79 \\
\hline \multicolumn{11}{|c|}{ IC 5152} \\
\hline Min.-disc & 44 & 10.0 & 34.26 & 1.80 & 4.92 & 44 & 10.0 & 31.03 & 1.90 & 4.51 \\
\hline Min.-disc + gas & 44 & 10.0 & 43.47 & 2.72 & 7.09 & 44 & 10.0 & 37.09 & 2.52 & 5.12 \\
\hline Max.-disc & 44 & 10.0 & 79.97 & 5.57 & 6.93 & 44 & 10.0 & 90.67 & 13.45 & 10.58 \\
\hline
\end{tabular}

Notes: (1) and (6): The rotation velocity at $r_{200}$, estimated from the flat part of the rotation curve; (2) and (7): the concentration parameter derived following Eq. (7); (3) and (8): the fitted virial radius; (4) and (9): uncertainties of $r_{200}$; (5) and (10): reduced $\chi^{2}$ values.

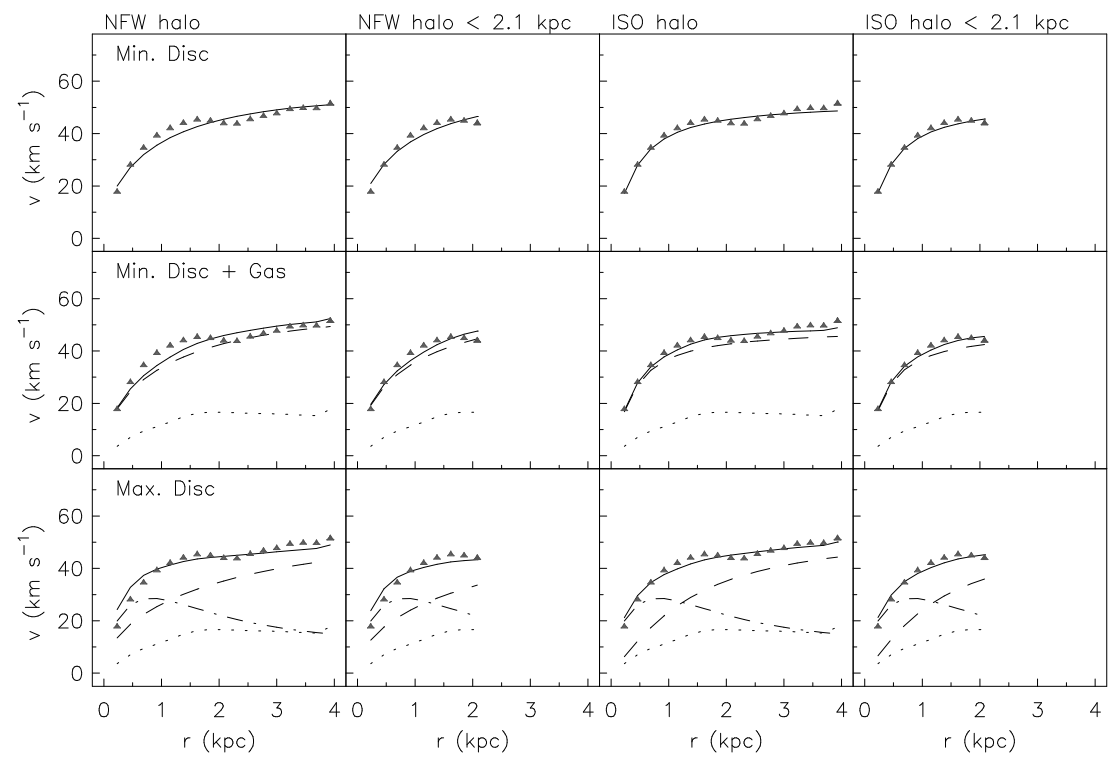

Fig. 15. Mass decomposition of IC 5152. The same as in Fig. 10. A reduced rotation curve is fitted up to a radius of $2.1 \mathrm{kpc}$.
Figure 14 shows the resulting fits. Again, the $\chi_{\text {red }}^{2}$ values are very high for the NFW halo and much smaller for the ISO halo. For this galaxy, the reduced rotation curve did not improve the quality of the models, which is probably due to the fact that the repeated rise of the rotation curve beyond a radius of $7 \mathrm{kpc}$ matches the first rise of the curve.

\subsection{IC 5152}

The results for IC 5152 are presented in Fig. 15. We fit NFW and ISO haloes to the entire radial range and to the inner $2.1 \mathrm{kpc}$ only. Although both dark matter halo models provide adequate fits (the $\chi_{\text {red }}^{2}$ values for the NFW model are the lowest of our 
Table 7. The basic parameters of the mass decomposition - the pseudo-isothermal halo model.

\begin{tabular}{|c|c|c|c|c|c|c|c|c|c|c|}
\hline \multirow[b]{2}{*}{$\begin{array}{l}\text { Parameter } \\
\text { [Unit] }\end{array}$} & \multicolumn{5}{|c|}{ ISO full } & \multicolumn{5}{|c|}{ ISO small } \\
\hline & $\begin{array}{c}\rho_{0} \\
{\left[10^{-3} M_{\odot} \mathrm{pc}^{-3}\right]} \\
(1)\end{array}$ & $\begin{array}{c}\Delta \rho_{0} \\
{\left[10^{-3} M_{\odot} \mathrm{pc}^{-3}\right]} \\
(2)\end{array}$ & $\begin{array}{c}r_{\mathrm{c}} \\
{[\mathrm{kpc}]} \\
(3) \\
\end{array}$ & $\begin{array}{c}\Delta r_{\mathrm{c}} \\
{[\mathrm{kpc}]} \\
(4)\end{array}$ & $\begin{array}{l}\chi_{\text {red }}^{2} \\
(5) \\
\end{array}$ & $\begin{array}{c}\rho_{0} \\
{\left[10^{-3} M_{\odot} \mathrm{pc}^{-3}\right]} \\
(6) \\
\end{array}$ & $\begin{array}{c}\Delta \rho_{0} \\
{\left[10^{-3} M_{\odot} \mathrm{pc}^{-3}\right]} \\
(7)\end{array}$ & $\begin{array}{c}r_{\mathrm{c}} \\
{[\mathrm{kpc}]} \\
(8) \\
\end{array}$ & $\begin{array}{c}\Delta r_{\mathrm{c}} \\
{[\mathrm{kpc}]} \\
(9) \\
\end{array}$ & $\begin{array}{l}\chi_{\mathrm{red}}^{2} \\
(10) \\
\end{array}$ \\
\hline \multicolumn{11}{|c|}{ NGC 2366} \\
\hline Min.-disc & 56.66 & 10.42 & 0.94 & 0.11 & 27.82 & 32.95 & 1.33 & 1.58 & 0.05 & 1.79 \\
\hline Min.-disc + gas & 53.12 & 14.46 & 0.77 & 0.13 & 30.27 & 27.54 & 1.02 & 1.44 & 0.04 & 0.88 \\
\hline Max.-disc & 25.28 & 9.82 & 0.92 & 0.22 & 24.99 & 11.27 & 0.72 & 2.23 & 0.14 & 1.24 \\
\hline \multicolumn{11}{|c|}{ ESO 059-G001 } \\
\hline Min.-disc & 67.08 & 3.18 & 1.48 & 0.06 & 0.84 & $\ldots$ & $\ldots$ & $\ldots$ & $\ldots$ & $\ldots$ \\
\hline Min.-disc + gas & 62.98 & 3.58 & 1.49 & 0.07 & 1.10 & $\ldots$ & $\ldots$ & $\ldots$ & $\ldots$ & $\ldots$ \\
\hline Max.-disc & 25.70 & 5.37 & 3.03 & 0.76 & 12.52 & $\ldots$ & $\ldots$ & $\ldots$ & $\ldots$ & $\ldots$ \\
\hline \multicolumn{11}{|c|}{ ESO 215-G?009 } \\
\hline Min.-disc & 77.74 & 10.73 & 1.27 & 0.11 & 18.42 & 71.67 & 9.27 & 1.35 & 0.12 & 15.67 \\
\hline Min.-disc + gas & 98.86 & 15.22 & 1.01 & 0.10 & 16.47 & 89.34 & 11.62 & 1.10 & 0.09 & 11.77 \\
\hline Max.-disc & 34.27 & 8.49 & 1.47 & 0.24 & 19.18 & 28.47 & 6.10 & 1.71 & 0.26 & 14.44 \\
\hline \multicolumn{11}{|c|}{ NGC 4861 } \\
\hline Min.-disc & 13.98 & 1.58 & 2.22 & 0.18 & 4.29 & 11.06 & 0.70 & 3.04 & 0.22 & 1.00 \\
\hline Min.-disc + gas & 9.67 & 1.29 & 2.45 & 0.24 & 3.91 & 8.75 & 0.98 & 2.90 & 0.35 & 1.72 \\
\hline Max.-disc & 4.85 & 1.13 & 3.93 & 0.81 & 10.40 & 3.95 & 1.48 & 7.10 & 7.19 & 14.21 \\
\hline \multicolumn{11}{|c|}{ NGC 5408} \\
\hline Min.-disc & 9.22 & 2.11 & 3.36 & 0.68 & 20.68 & 6.69 & 0.87 & 6.37 & 1.66 & 6.85 \\
\hline Min.-disc + gas & 6.98 & 1.76 & 3.70 & 0.87 & 18.13 & 4.94 & 0.78 & 8.64 & 4.05 & 7.24 \\
\hline \multicolumn{11}{|c|}{ IC 5152} \\
\hline Min.-disc & 378.79 & 40.76 & 0.37 & 0.02 & 1.94 & 389.45 & 40.23 & 0.36 & 0.02 & 1.24 \\
\hline Min.-disc + gas & 369.69 & 48.16 & 0.34 & 0.03 & 2.23 & 398.06 & 49.18 & 0.33 & 0.03 & 1.36 \\
\hline Max.-disc & 42.11 & 6.70 & 1.18 & 0.14 & 2.87 & 47.24 & 15.81 & 1.08 & 0.36 & 4.44 \\
\hline
\end{tabular}

Notes: (1) and (6): Fitted core density $\rho_{0}$ of the pseudo-isothermal halo model; (2) and (7): uncertainties of $\rho_{0}$; (3) and (8): fitted core $r_{\mathrm{c}}$ radius of the pseudo-isothermal halo model; (4) and (9): uncertainties of $r_{\mathrm{c}} ;(5)$ and (10): reduced $\chi^{2}$ values.

sample), the ISO model still gives better values than the NFW halo.

\subsection{Discussion}

In contrast to recent work by $\mathrm{dB} 08$ and $\mathrm{Oh} 08$, we fitted the NFW profiles with fixed values of $v_{200}$ and $c$ (see the beginning of this section). With this approach we assumed reasonable values for $v_{200}$ and $c$ that follow cosmological predictions, and also obtained reasonable results for $r_{200}$. Nevertheless, we could show that in general, the observed rotation curves can be better fitted by an ISO halo than by an NFW halo, although at least in the case of ESO 215-G?009 the NFW fits are equally good or even better than the ISO fits. The values of the core radius $r_{\mathrm{c}}$ and the core density $\rho_{0}$ are plausible and in good agreement with results for LSB galaxies (de Blok et al. 2001a; Kuzio de Naray et al. 2008) and for dwarf galaxies (Spano et al. 2008). They also follow the recent finding of a constant central surface density $\mu_{0 \mathrm{D}}=r_{\mathrm{c}} \rho_{0}$ (Donato et al. 2009, and references therein).

As mentioned in the introduction, CDM simulations predict density profiles with a steep inner slope of $\alpha=-1$ (Navarro et al. 1996) or even $\alpha=-1.5$ (Moore et al. 1998). de Blok et al. (2001a) showed for their sample of LSB galaxies that the observed distribution of $\alpha$ peaks at $-0.2 \pm 0.2$. In order to compare these results with ours, we first measure the slopes in the inner $\mathrm{kpc}$ of our data by plotting the logarithm of the density vs. the logarithm of the radius (see Fig. 16). For all galaxies except for IC 5152, $\alpha$ lies between -0.43 and 0.03 (see also Table 8), which is within the errors of the $\alpha$ determined by de Blok et al. (2001a).
ESO 215-G?009, which partly showed higher $\chi_{\text {red }}^{2}$ values for the ISO halo model than for the NFW halo model (see Sect. 6.3), has an $\alpha$ of -0.35 . This means that its innermost slope can better be described by an ISO halo.

In Fig. 16 we also fitted the ISO and NFW profiles of the minimum-disc case (dotted and long-dashed lines, respectively). They do not always agree with the observed density profiles, which is to be expected as $\chi_{\text {red }}^{2}$ is often quite high, indicating a bad fit.

Figure 17 shows $\alpha$ vs. the logarithm of the innermost radius (de Blok et al. 2001a, see also Oh08). Data from previous studies are plotted with grey open circles (de Blok et al. 2001a), grey open squares (de Blok \& Bosma 2002), grey open triangles (Swaters et al. 2003), and black solid triangles (Oh08). Our own results are plotted with black solid squares and are in good agreement with all previous works. Only IC 5152, which has an inner slope of $\alpha=-0.82$, is close to CDM predictions.

We now discuss possible systematic errors in the data: Swaters et al. (2003) and Spekkens et al. (2005) used rotation curves of dwarf and low surface brightness galaxies obtained from optical long-slit spectra in order to measure the slopes of the mass distribution. They compared their results to model spectra by taking into account possible sources of uncertainties like slit misalignment, slit width, and seeing. Both studies came to the result that the galaxies do not require haloes with steep cusps, but that haloes with $\alpha=1$ cannot be ruled out. However, they clearly showed that a steeper slope $(\alpha>1)$ is not consistent with the observed profiles. As we use 2D velocity fields, our data are not affected by the abovementioned errors. We can also 


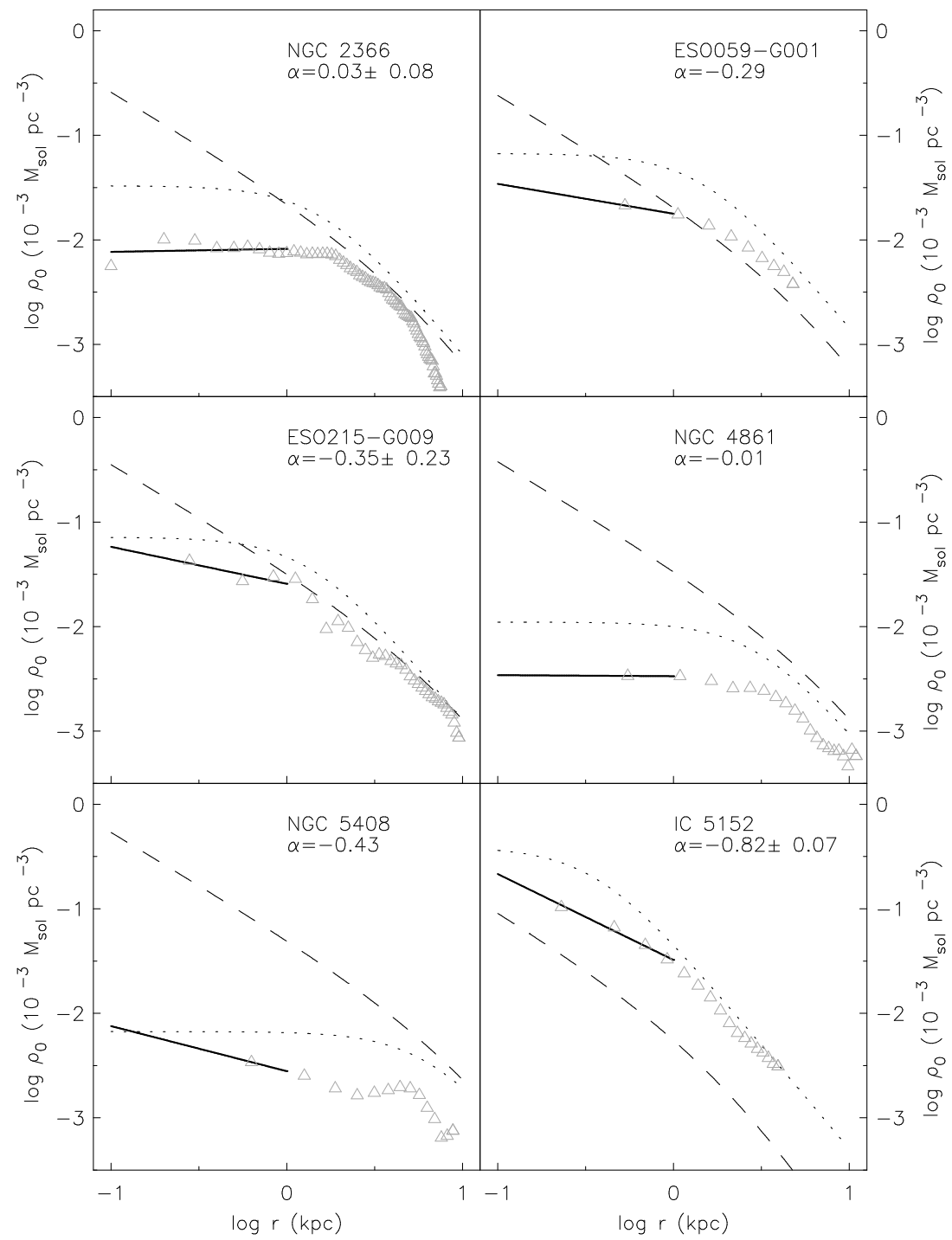

Fig. 16. The density profiles of all sample galaxies derived from the observed rotation curves (open grey triangles). Their inner slopes $\alpha$ are measured by applying a least square fit to all data points within the innermost kpc (bold black lines). The fitted values of $\alpha$ and the uncertainties are placed into the upper right corner of each panel. Note that the rotation curves of ESO 059-G001, NGC 4861, and NGC 5408 only contain two points in the inner $1 \mathrm{kpc}$. Therefore, no uncertainties can be given. The long-dashed and dotted lines show the NFW and the ISO profiles, respectively, using the parameters of the minimum-disc case.
Table 8. Slope $\alpha$ in the inner kpc.

\begin{tabular}{lccc}
\hline \hline Galaxy & $\alpha$ & $\Delta \alpha$ & $\begin{array}{c}r_{\text {in }} \\
{[\mathrm{kpc}]} \\
(3)\end{array}$ \\
\hline NGC 2366 & 0.03 & 0.08 & 0.10 \\
ESO 059-G001 & -0.29 & $\ldots$ & 0.52 \\
ESO 215-G?009 & -0.35 & 0.23 & 0.28 \\
NGC 4861 & -0.01 & $\ldots$ & 0.50 \\
NGC 5408 & -0.43 & $\ldots$ & 0.35 \\
IC 5152 & -0.82 & 0.07 & 0.23 \\
\hline
\end{tabular}

Notes: (1) The slope of the inner $1 \mathrm{kpc}$ of the density profile; (2) uncertainties; (3) the radius of the innermost point.

rule out geometric effects caused by low velocity gas picked up along the line of sight at higher inclinations, as our data have a very high spectral resolution.

As already mentioned in Sect. 4.1, CT08 could show that inaccurate centre positions do not change the inner slope of a rotation curve. Non-circular motions are detected, but low enough to not significantly change the rotation velocities. Nevertheless, there are problems that affect our results such as the low spatial resolution, especially of the ATCA data.

Thus, for the majority of galaxies studied here, the ISO halo reproduces the observed rotation curves much better than the NFW halo, which is in good agreement with previous studies (e.g., Kuzio de Naray et al. 2008; Spano et al. 2008). The slopes of the observed rotation curves are inconsistent with CDM predictions, but agree with the results from de Blok et al. (2001a). One explanation for the cusp-core discrepancy might be that most simulations neglect baryons. And indeed, first approaches to include baryons into CDM simulations show that their contribution changes the inner density profile from a cusp to a core (Romano-Díaz et al. 2008).

\section{Summary}

We used VLA and ATCA H I synthesis data of a sample of six nearby irregular dwarf galaxies in order to decompose the observed rotation curves into the contributions from stars, gas, and the dark matter halo. In order to rule out systematic effects, we first performed a harmonic decomposition of the Hermite velocity fields. The results show that the quadratically-added amplitude $A_{\mathrm{r}}(r)$ is generally below $6 \mathrm{~km} \mathrm{~s}^{-1}$, in the central kpc it is even below $3 \mathrm{~km} \mathrm{~s}^{-1}$. Over the entire radial range, non-circular 


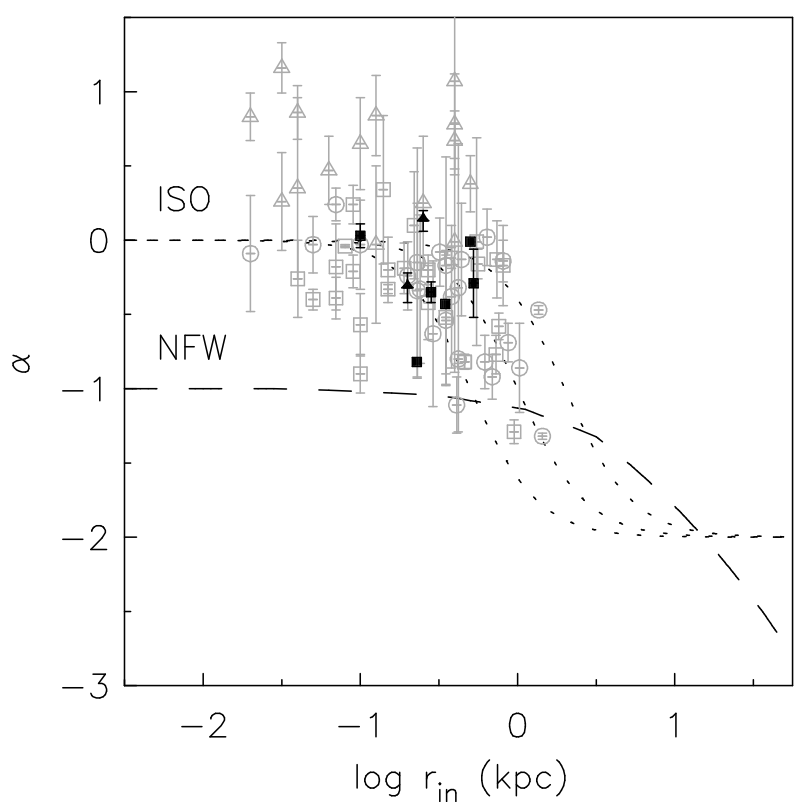

Fig. 17. The inner slope of the dark matter density profiles plotted against the radius of the innermost point. Presented in grey are the results from publications by de Blok et al. (2001a) (open circles), de Blok \& Bosma (2002) (open squares), and by Swaters et al. (2003) (open triangles). Shown in black are the results from $\mathrm{Oh} 08$ (solid triangles). Our results are overplotted with black squares. We fit the isothermal and NFW profiles following de Blok et al. (2001a).

motions contribute less than $25 \%$, sometimes even less than $10 \%$ to the local rotation velocity (constrained case). According to Hayashi et al. (2004), the triaxial dark matter haloes cause noncircular motions as large as $50 \%$ of the local rotation velocity within the central kpc. Our much smaller amplitudes demonstrate that the observed rotation curves of the galaxies studied here are not severely influenced by non-circular motions.

With this knowledge, we decomposed the observed rotation curves into their components. We fitted a cuspy NFW halo and a cored pseudo-isothermal halo to the rotation curves and modelled the minimum-disc, the minimum-disc + gas, and the maximum-disc cases. The resulting model fits show that in all sample galaxies (except for ESO 215-G?009), the observed rotation curves are better represented by the ISO halo than by the NFW halo. As the outer parts of the rotation curves are affected by uncertainties caused by the sparsely filled tilted rings, we also performed a mass decomposition by fitting the inner few kpcs only. For most galaxies, this significantly improved the quality of both the NFW and the ISO halo models. The reduced $\chi^{2}$ could in most cases be decreased for both halo models by including the gas. In some cases, a further improvement could be achieved when also including the stars. This implies that baryons should not be neglected, at least not in the inner part of dwarf galaxies (see also Swaters et al. 2009).

Acknowledgements. The authors would like to thank the anonymous referee for the useful comments that significantly improved this paper.

Furthermore, we thank Nic Bonne and Erwin de Blok for their help with the mass decomposition. We would also like to thank Fabian Walter for providing the THINGS data of NGC 2366 and Eric Wilcots for providing the VLA data cubes of NGC 4861.

This work was partly supported by the Deutsche Forschungsgesellschaft (DFG) under the SFB 591, by the Research School of the Ruhr-Universität Bochum, and by the Australia Telescope National Facility, CSIRO. It is partly based on observations with the Australia Telescope Compact Array. The ATCA is part of the Australia Telescope which is funded by the Commonwealth of Australia for operation as a National Facility managed by CSIRO. It is also partly based on archival VLA data of the National Radio Astronomy Observatory. The NRAO is a facility of the National Science Foundation operated under cooperative agreement by Associated Universities, Inc.

We made use of NASA's Astrophysics Data System (ADS) Bibliographic Services and the NASA/IPAC Extragalactic Database (NED) which is operated by the Jet Propulsion Laboratory, California Institute of Technology, under contract with the National Aeronautics and Space Administration.

\section{References}

Binney, J., \& Tremaine, S. 1987, Galactic Dynamics (Princeton, NJ: Princeton University Press), 747

Bosma, A. 1978, Ph.D. Thesis, Groningen Univ.

Côté, S., Carignan, C., \& Freeman, K. C. 2000, AJ, 120, 3027

de Blok, W. J. G. 2004, in Dark Matter in Galaxies, ed. S. Ryder, D. Pisano,

M. Walker, \& K. Freeman, IAU Symp., 220, 69

de Blok, W. J. G., \& Bosma, A. 2002, A\&A, 385, 816

de Blok, W. J. G., \& McGaugh, S. S. 1997, MNRAS, 290, 533

de Blok, W. J. G., McGaugh, S. S., Bosma, A., \& Rubin, V. C. 2001a, ApJ, 552, L23

de Blok, W. J. G., McGaugh, S. S., \& Rubin, V. C. 2001b, AJ, 122, 2396

de Blok, W. J. G., Bosma, A., \& McGaugh, S. 2003, MNRAS, 340, 657

de Blok, W. J. G., Walter, F., Brinks, E., et al. 2008, AJ, 136, 2648

de Vaucouleurs, G., de Vaucouleurs, A., Corwin, H. G., et al. 1991, Third

Reference Catalogue of Bright Galaxies (Vols. 1-3, XII, Berlin-Heidelberg-

New York: Springer-Verlag)

Donato, F., Gentile, G., Salucci, P., et al. 2009 [arXiv: e-prints]

Flores, R. A., \& Primack, J. R. 1994, ApJ, 427, L1

Gentile, G., Salucci, P., Klein, U., Vergani, D., \& Kalberla, P. 2004, MNRAS, 351,903

Gentile, G., Burkert, A., Salucci, P., Klein, U., \& Walter, F. 2005, ApJ, 634, L145 Gil de Paz, A., \& Madore, B. F. 2005, ApJS, 156, 345

Hayashi, E., \& Navarro, J. F. 2006, MNRAS, 373, 1117

Hayashi, E., Navarro, J. F., Jenkins, A., et al. 2004 [arXiv:Astrophysics e-prints] Hunter, D. A., Elmegreen, B. G., \& van Woerden, H. 2001, ApJ, 556, 773

Karachentsev, I. D., Sharina, M. E., Dolphin, A. E., et al. 2002a, A\&A, 385, 21

Karachentsev, I. D., Sharina, M. E., Makarov, D. I., et al. 2002b, A\&A, 389, 812

Karachentsev, I. D., Dolphin, A., Tully, R. B., et al. 2006, AJ, 131, 1361

Karachentsev, I. D., Tully, R. B., Dolphin, A., et al. 2007, AJ, 133, 504

Kirby, E. M., Jerjen, H., Ryder, S. D., \& Driver, S. P. 2008, AJ, 136, 1866

Kirby, E. M., et al. 2009, in preparation

Koribalski, B. S., \& Jerjen, H. 2008, in Galaxies in the Local Volume, ed. B. S.

Koribalski, \& H. Jerjen (Berlin: Springer), ISBN 978-1-4020-6932-1

Koribalski, B. S., et al. 2009, in preparation

Kuzio de Naray, R., McGaugh, S. S., de Blok, W. J. G., \& Bosma, A. 2006, ApJS, 165, 461

Kuzio de Naray, R., McGaugh, S. S., \& de Blok, W. J. G. 2008, ApJ, 676, 920

Leroy, A., Bolatto, A. D., Simon, J. D., \& Blitz, L. 2005, ApJ, 625, 763

Marchesini, D., D’Onghia, E., Chincarini, G., et al. 2002, ApJ, 575, 801

Moore, B., Governato, F., Quinn, T., Stadel, J., \& Lake, G. 1998, ApJ, 499, L5

Moore, B., Quinn, T., Governato, F., Stadel, J., \& Lake, G. 1999, MNRAS, 310, 1147

Navarro, J. F., Frenk, C. S., \& White, S. D. M. 1996, ApJ, 462, 563

Noeske, K. G., Papaderos, P., Cairós, L. M., \& Fricke, K. J. 2003, A\&A, 410, 481

Oh, S.-H., de Blok, W. J. G., Walter, F., Brinks, E., \& Kennicutt, R. C. 2008, AJ, 136, 2761

Parodi, B. R., Barazza, F. D., \& Binggeli, B. 2002, A\&A, 388, 29

Portinari, L., Sommer-Larsen, J., \& Tantalo, R. 2004, MNRAS, 347, 691

Rhee, G., Valenzuela, O., Klypin, A., Holtzman, J., \& Moorthy, B. 2004, ApJ, 617,1059

Romano-Díaz, E., Shlosman, I., Hoffman, Y., \& Heller, C. 2008, ApJL, 685, L105

Rubin, V. C., Thonnard, N., \& Ford, Jr., W. K. 1978, ApJ, 225, L107

Salucci, P. 2001, MNRAS, 320, L1

Schoenmakers, R. H. M. 1999, Ph.D. Thesis, Rijksuniversiteit Groningen, The Netherlands

Schoenmakers, R. H. M., Franx, M., \& de Zeeuw, P. T. 1997, MNRAS, 292, 349 Sellwood, J. A., \& McGaugh, S. S. 2005, ApJ, 634, 70

Sersic, J. L. 1968, Atlas de galaxias australes (Cordoba, Argentina: Observatorio Astronomico)

Simon, J. D., Bolatto, A. D., Leroy, A., Blitz, L., \& Gates, E. L. 2005, ApJ, 621, 757

Spano, M., Marcelin, M., Amram, P., et al. 2008, MNRAS, 383, 297

Spekkens, K., \& Sellwood, J. A. 2007, ApJ, 664, 204

Spekkens, K., Giovanelli, R., \& Haynes, M. P. 2005, AJ, 129, 2119 
Spergel, D. N., Verde, L., Peiris, H. V., et al. 2003, ApJS, 148, 175

Spergel, D. N., Bean, R., Doré, O., et al. 2007, ApJS, 170, 377

Springel, V., White, S. D. M., Jenkins, A., et al. 2005, Nature, 435, 629

Swaters, R. A., Madore, B. F., van den Bosch, F. C., \& Balcells, M. 2003, ApJ, 583,732

Swaters, R. A., Sancisi, R., van Albada, T. S., \& van der Hulst, J. M. 2009, A\&A, 493, 871

Taylor, C. L., Kobulnicky, H. A., \& Skillman, E. D. 1998, AJ, 116, 2746

Thuan, T. X., Hibbard, J. E., \& Lévrier, F. 2004, AJ, 128, 617

Tikhonov, N. A., \& Galazutdinova, O. A. 2008, Astron. Rep., 52, 19

Tolstoy, E., Saha, A., Hoessel, J. G., \& McQuade, K. 1995, AJ, 110, 1640

Trachternach, C., de Blok, W. J. G., Walter, F., Brinks, E., \& Kennicutt, Jr., R. C. 2008, AJ, 136, 2720 van den Bosch, F. C., Robertson, B. E., Dalcanton, J. J., \& de Blok, W. J. G. 2000, AJ, 119, 1579

van der Hulst, J. M., Terlouw, J. P., Begeman, K. G., Zwitser, W., \& Roelfsema,

P. R. 1992, in Astronomical Data Analysis Software and Systems I, ed. D. M. Worrall, C. Biemesderfer, \& J. Barnes, ASP Conf. Ser., 25, 131

van Eymeren, J. 2008, Ph.D. Thesis, Astronomisches Institut der RuhrUniversitaet Bochum, Germany

van Eymeren, J., Marcelin, M., Koribalski, B., et al. 2009a, A\&A, 493, 511 van Eymeren, J., Marcelin, M., Koribalski, B. S., et al. 2009b, A\&A, 505, 105 Walter, F., Brinks, E., de Blok, W. J. G., et al. 2008, AJ, 136, 2563

Warren, B. E., Jerjen, H., \& Koribalski, B. S. 2004, AJ, 128, 1152

Warren, B. E., Jerjen, H., \& Koribalski, B. S. 2006, AJ, 131, 2056

Wilcots, E. M., Lehman, C., \& Miller, B. 1996, AJ, 111, 1575 